\title{
NON-ACCELERATOR PARTICLE PHYSICS
}

\author{
Progress Report for Period \\ February 15, 1991 - September 15, 1991 \\ R.I. Steinberg and C.E. Lane \\ Drexel University \\ Department of Physics \\ Philadelphia, PA 19104 \\ September 1991
}

\begin{abstract}
NOTICE
This report was prepared as an account of work sponsored by the United States Government. Neither the United States nor the Department of Energy, nor any of their employees. nor any of their contractors, subcontractors, or their employees, makes any warranty, express or implied, or assumes any legal liability or responsibility for the accuracy, completeness, or usefulness of any information, apparatus, product or process disclosed or represents that its use would not infringe privately-owned rights.
\end{abstract}

Prepared for:

THE U. S. DEPARTMENT OF ENERGY

Contract No. DE-FG02-91ER40615 


\section{Contents}

1 Introduction 2

2 The MACRO experiment 6

2.1 MACRO liquid scintillator ............. 6

2.1 .1 Design . . . . . . . . . . . . . 6

2.1 .2 Production and testing . . . . . . . . . 7

2.2 MACRO fast monopole electronics . . . . . . . . . 8

2.2 .1 Introduction . . . . . . . . . . . . 8

2.2.2 MACRO fast monopole physics . . . . . . . . 8

2.2 .3 Trigger design .................... 10

2.2 .4 Implementation . . . . . . . . . . . . . 15

2.3 MACRO data analysis . . . . . . . . . . . . 23

2.4 Shifts and on-site work ............... 24

3 Search for neutrino oscillations $\mathbf{2 5}$

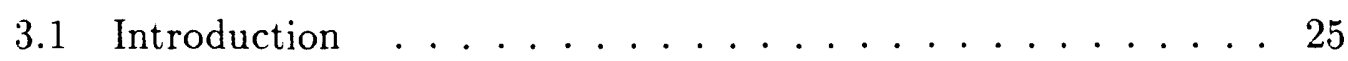

3.2 Experimental searches for neutrino mass . . . . . . . . 25

3.3 Neutrino vacuum flavor oscillations ........... . . 25

3.4 Reactor neutrino oscillation experiments . . . . . . . . 26

4 The Perry experiment 2

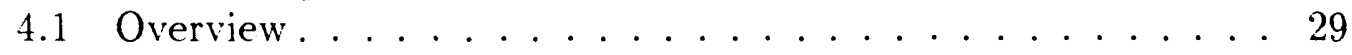

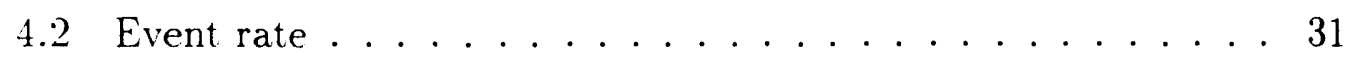

4.3 Backgrounds ........................... 32

4.4 A chlorine-loaded neutron capture scintillator ....... 34

4.4 .1 Introduction . . . . . . . . . . . . . 34

4.4 .2 Performance goals . . . . . . . . . . . . . 35

4.4.3 Scintillation yield and optical transparency . . . . . . 36

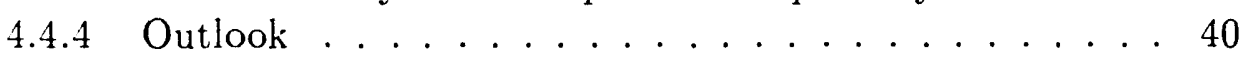

4.5 Event generation and reconstruction .......... . . 41

4.6 In situ background tests ................ 44

5 Drexel Particle Physics Publications, 1991

$\begin{array}{ll}\text { References } & \mathbf{4 7}\end{array}$ 


\section{List of Figures}

1 Astrophysical and experimental bounds on the monopole flux . 9

2 Expected scintillation yield for a magnetic monopole. . . . . . 9

3 Experimental signature of a monopole . . . . . . . . . . . 10

4 Comparison of signatures in MACRO . . . . . . . . . 11

5 Fast monopole trigger logic . . . . . . . . . . . . . 13

6 Coverage of the triggers ............... . . 14

7 Schematic for the fast linear $8: 1$ fanin . . . . . . . . . . 17

8 Response function of production fanin . . . . . . . . . 18

9 Response function of 8 inputs of fanin . . . . . . . . 18

10 Partial schematic for the plane logic module . . . . . . . . 20

11 Calibration and testing scheme for fast monopole triggers . . . 22

12 Exclusion plot................. . . 28

13 The Perry underground neutrino oscillation experiment . . . 30

14 Chlorinated scintillator pulse height spectra . . . . . . . 38

15 Chlorinated scintillator pulse height spectra . . . . . . . 38

16 Scintillation efficiency of 1,2,3-trichloropropane scintillators . . 39

17 Optical attenuation for 1,2,3-trichloropropane . . . . . . . . 39

18 Ray trace for events in scintillator sphere . . . . . . . . . . . 42

19 Reconstruction of $1008-\mathrm{MeV}$ point events . . . . . . . . 43

\section{List of Tables}

1 Inverse beta decay event rate in the Perry experiment . . . . . 31

2 Comparison of neutron capture scintillators . . . . . . . 35

3 Performance goals for the Perry scintillator . . . . . . . . . 35

4 Candidate materials for chlorinated scintillators . . . . . . 36

$5 \quad$ Chlorobenzene (CB) scintillator efficiency . . . . . . . . . 37

6 Input parameters for point event reconstruction program . . . 41 


\begin{abstract}
The goals of this research are the experimental testing of fundamental theories of physics such as grand unification and the exploration of cosmic phenomena through the techniques of particle physics. We are working on the MACRO experiment, which employs a large area underground detector to search for grand unification magnetic monopoles and dark matter candidates and to study cosmic ray muons as well as low and high energy neutrinos; the $\nu$ IMB project, which seeks to refurbish and upgrade the IMB water Cerenkov detector to perform an improved proton decay search together with a long baseline reactor neutrino oscillation experiment using a one kiloton liquid scintillator (the Perry experiment); and development of technology for improved liquid scintillators and for very low background materials in support of the MACRO and Perry experiments and for new solar neutrino experiments.
\end{abstract}




\section{Introduction}

This document is a progress report for the Drexel University-United States Department of Energy contract supporting research in experimental particle physics. Our research is in the following areas:

- the MACRO experiment, which is employing a large area underground detector to search for grand unification magnetic monopoles and dark matter candidates and to study cosmic ray muons as well as low and high energy neutrinos;

- the $\nu$ IMB project, which seeks to refurbish and upgrade the IMB water Cerenkov detector to perform an improved proton decay search together with a long baseline reactor neutrino oscillation experiment using a one kiloton liquid scintillator (the Perry experiment):

As described in Section 2, construction of the MACRO detector in Hall B at the Gran Sasso underground laboratory (Abruzzi, Italy), has made rapid progress, with the lower part of six supermodules now completed. The first supermodule has been run for over a year of live time and has produced a substantial data set from which a significant number of new physics results were obtained.

Over 1.5 million muons have been recorded with MACRO, and an extensive analysis of the early (Spring '89) runs has been especially fruitful. Preliminary results were obtained on the vertical muon flux, the lateral spread and multiplicity distributions of muon bundles, a search for Grand Unified Theory magnetic monopoles, and a search for electron antineutrinos from stellar collapses, as well as surface-underground coincidences between MACRO and EASTOP (an extensive air shower array located on the mountain above MACRO and operated by a group from Turin University).

Preliminary results from the analysis of Spring ' 89 run data were presented at the International Cosmic Ray Conference in Adelaide, Australia in early 1990 [1], and at talks given at the Moriond Workshop [2] and the American Physical Society conference in Washington D.C. [3]. In addition, a paper presenting a more complete analysis of single-muon physics has been published [4]. together with a paper on observation of surface-underground coincidences $[5]$. 
New results are being presented at the Dublin International Co mic Ray Conference on charmed hadro-production [6], the muon decohererce function [7]. muon point sources [8], muon anisotropies and time varistions [9], stellar gravitational collapse [10], a flux limit for nuclearites [11], and the primary cosmic ray composition [12]. We also expect a paper on monopole flux limits to be ready for publication in the next few months.

Three U.S. MACRO students have recently graduated with theses based on analyses of MACRO data from the Spring ' 89 run $[13,14,15]$. These theses are important to the collaboration, because they contain the type of careful in-depth analysis necessary for a complete understanding of the experiment. This process is continuing with the more recent data, ans one or two more theses are expected in the coming year.

The first supermodule of MACRO continues to run while the remai der of the experiment is being instrumented and tested. Part of the new instrumentation being installed and tested on MACRO is a fast monopole and global muon trigger, developed at Drexel by Prof. Lane. This trigger will give MACRO sensitivity to monopoles with velocity $\beta>10^{-3}$, as well as being useful for triggering on fractionally charged particles. In late 1991 or early 1992. after supermodules 2 through 6 are operational, the first supermodule will be shut down and re-instrumented using the new electronics.

The outstanding performance of the MACRO experiment has been mads possible to a large extent by our development of high transparency liquid scintillators.

At present. more than 500,000 liters of the Drexel scintillator have been installed in MACRO. The performance of the scintillator in the detector is frlly consistent with that obtained during development testing at Drexel. The attenuation length (the most critical parameter for the 12-meter MACRO detector modules) is about $12 \mathrm{~m}$, including losses both from the liquid scintillator itself and from the toially reflecting teflon liner of the detector boxes. This high transparency means that the ratio of light collected from the near end of a module to that from the far end is only about $4: 1$. At the center of a tank. a minimum ionizing particle produces approximately 400 photoelectrons. while at a distance of 11 meters, such a particle yields 240 photoelectrons. No other detector of similar geometry has attained this level of performance. 
Beyond the continuing work of producing and testing the MACRO scintillator, we have constructed trigger and calibration electronics as well as participated in the physics analysis of the MACRO data stream. For this work our group has developed the capability for design, construction and testing of fast trigger electronics, which should also prove useful for other experiments.

As the second major project for our group, we have submitted to the Department of Energy, together with the present members of the IMB collaboration, a letter of intent to perform a major new highly sensitive longbaseline deep-underground reactor neutrino oscillation experiment (the Perry experiment, Section 4). The new experiment will take advantage of existing equipment and experience at the IMB Fairport (Ohio) underground facility and will use antineutrinos from the $3600 \mathrm{MW}$ Perry Nuclear Power Reactor of the Cleveland Electric Illuminating Co., optimally situated at a distance of $12.9 \mathrm{~km}$ from the underground laboratory.

The goal of the Perry experiment is to gain a factor of 200 improvement in our knowledge of the mass difference squared parameter, $\Delta m^{2}$, that ccntrols neutrino oscillations, thereby providing a sensitive new probe for finite neutrino masses. For large mixing angles, the experiment will detect oscillations for values of $\Delta m^{2}>8 \times 10^{-5} \mathrm{eV}^{2}$.

The concept of the experiment is to suspend a $12 \mathrm{~m}$-diameter transparent spherical acrylic container in the center of the IMB swimming pool. About 900 tons of a highly transparent chlorinated liquid scintillator (under development at Drexel) with a density slightly greater than that of water (to reduce stresses on the acrylic vessel) will permit efficient and nearly background free detection of the positrons and neutrons resulting from the inverse beta decay reaction of the $\bar{\nu}_{\epsilon}$ 's on the hydrogen atoms of the scintillator. Scintillation photons from particle interactions in the vessel will be collected by the existing 2048 eight-inch photomultipliers and processed by zero deadtime multi-hit adc/tdc's.

The 8000 tons of ultra-pure water in the IMB facility will serve both as passive shielding against ambient radioactivity and as active shielding against cosmic ray muon-induced backgrounds, which already will have been strongly suppressed by the 600 meters of rock overburden.

The $\nu$ IMB collaboration, formed to carry out this experiment, has as its initial goal to refurbish and upgrade the IMB detector to perform an 
improved proton decay search; at the same time we will continue the Drexelcentered development program for the Perry experiment with the aims of demonstrating feasibility of the experiment and developing a proposal within a year.

This progress report concludes with a list of our 1991 publications (Section 5). 


\section{The MACRO experiment}

The MACRO experiment [16] is a joint U.S.-Italian effort to construct and operate a magnetic monopole and cosmic ray muon and neutrino detector of unprecedented sophistication and sensitivity. The construction of the lower part of MACRO, which consists of six 'supermodules' $(12 \mathrm{~m} \times 12 \mathrm{~m} \times 4.8 \mathrm{~m}$ each), was completed in the Spring of 1991, with instrumentation of these supermodules currently underway. We expect to start physics runs with all six supermodules near the end of 1991.

The upper part of MACRO (the 'attico') has recently been approved by the Italian authorities. When the attico is completed, MACRO will have dimensions of $72 \mathrm{~m} \times 12 \mathrm{~m} \times 9 \mathrm{~m}$, with an acceptance for isotropic particle fluxes of $10,000 \mathrm{~m}^{2} \mathrm{sr}$.

\subsection{MACRO liquid scintillator}

Full implementation of the MACRO detector will require a total of almost one million liters of liquid scintillator. Since no commercial scintillator was available with sufficient transparency to give satisfactory performance in a detector such as MACRO with its 12-meter light collection path, our group took the responsibility of developing for MACRO a special high-transparency liquid scintillator.

\subsubsection{Design}

As a result of research in the scintillation laboratory at Drexel University, we succeeded in designing a new liquid scintillator (now widely known as -MAC'RO scintillator') with outstanding transparency (greater than 12 meters attenuation length for scintillation light) and good energy conversion efficiency (about $40 \%$ that of anthracene. the most efficient known organic scintillator). Furthermore. in collaboration with our MACRO colleagues at Indiana Iniversity and the University of Michigan, we have produced the MACRO scintillator in large quantities at a cost of about $\$ 1.25 /$ liter. For comparison. commercial liquid scintillators, even in large quantities, have been quoted to us at prices exceeding $\$ 2.00 /$ liter. We are therefore providing for $\mathrm{MACRO}$ a superior product at a saving of $\$ .75 /$ liter. For the million-liter finished detector. the total saving will be about $5750 \mathrm{~K}$. 


\subsubsection{Production and testing}

The process of producing the MACRO scintillator begins with preparation of scintillator concentrate at Drexel, together with direct shipment of previously tested and approved pseudocumene and mineral oil from their respective refineries to a liquid scintillator storage and blending facility at the Frascati laboratory near Rome, where the final mixing of the components is performed. The finished liquid scintillator is then transported to the Gran Sasso laboratory for installation in the MACRO detector.

This procedure allows the most critical steps to take place in the U.S. under the direct supervision of physicists. Testing of all ingredients (mineral oil. pseudocumene, PPO, bis-MSB, and stabilizers) and shipping containers (drums, Iso-tanks, etc.) and the critical step of producing the liquid scintillator concentrate therefore all take place with good quality control.

The extra shipping costs associated with scintillator concentrate production at Drexel are negligible compared with savings resulting from decreased risk of using substandard ingredien s, contamination, error, or other failure. Only the scintillator concentrate ingredients $(<1 \%$ of the finished scintillator) are subject to the extra step of transport from the various manufacturers to our mixing facility in Philadelphia before being shipped on to Italy.

At Drexel University we have currently produced 4,400 liters of liquid scintillator concentrate. The concentrate is shipped in nitrogen-blanketed drums from Philadelphia to Italy. In addition, we have provided 22,000 liters of pure scintillation grade pseudocumene for the experiment. These ingredients. when mixed with mineral oil, are sufficient for 730,000 liters of finished scintillator. As in prior years, the mineral oil we are using was produced from Alberta crude oil drawn from the trans-Canada pipeline, refined in Perth Amboy, New Jersey and shipped in containerized 20,000-liter Iso-tanks to Frascati.

Rigorous quality control tests at each step in the preparation of the MACRO scintillator assure that all components meet our specifications. The quality control tests consist of analysis of pre-shipment samples from each batch of mineral oil, pseudocumene, and scintillation fluors. The entire production operation is proceeding smoothly, on time and under budget, and includes stringent personnel and fire safety precautions. 


\subsection{MACRO fast monopole electronics}

\subsubsection{Introduction}

Magnetic monopoles are predicted in many varieties of Grand Unified Theories (G'T's). The GUT monopole should be supermassive $\left(M \sim 10^{16} \mathrm{GeV}\right)$ and would be found only in cosmic rays as a remnant of the high-temperature processes in the early universe.

One of the major objectives for the MACRO experiment is to detect GUT monopoles, or to set limits on them at a level which are low enough to exclude monopoles as a significant fraction of the 'missing mass' of the universe.

The Particle Physics group at Drexel is implementing the Fast Monopole (FASTM) trigger for MACRO, with the goal of detecting monopoles with velocities $\beta \gtrsim 2.5 \times 10^{-3}$. The FASTM trigger is implemented jointly with a 'global' fast particle trigger, since most of the triggering electronics is the same.

\subsubsection{MACRO fast monopole physics}

In the next few years, MACRO will be able to set new limits on magnetic monopole fluxes well below the Parker bound [17] for monopoles with $10^{-4}<$ $3<1$ (see Fig. 1). Current limits on 'fast' monopoles $\left(\beta \gtrsim 10^{-2}\right)$ are already below the Parker bound; however, MACRO should be able to place limits at least a factor of ten below other monopole search limits over a broad range of monopole velocities.

The ability to detect GUT magnetic monopoles in MACRO hinges upon the scintillation light yield of a monopole passing through MACRO's liquid scintillation counters. This light yield has been calculated using experimental data for the scintillation yield of slow protons $[18,19]$, and is shown as a function of monopole velocity $\beta$ in Fig. 2.

Since monopoles are expected to be travelling at $\beta<<1$, this scintillation light is spread out over the transit time of the monopole through the counter, and gives a distinctive 'square' experimental signature (see Fig. 3). Fast monopoles have a less distinctive pulse, since the transit time through the scintillation counters is much less than that of slow monopoles. The time diffe:ence between the signals from monopole entry and exit, however, is still a very useful experimental handle. 


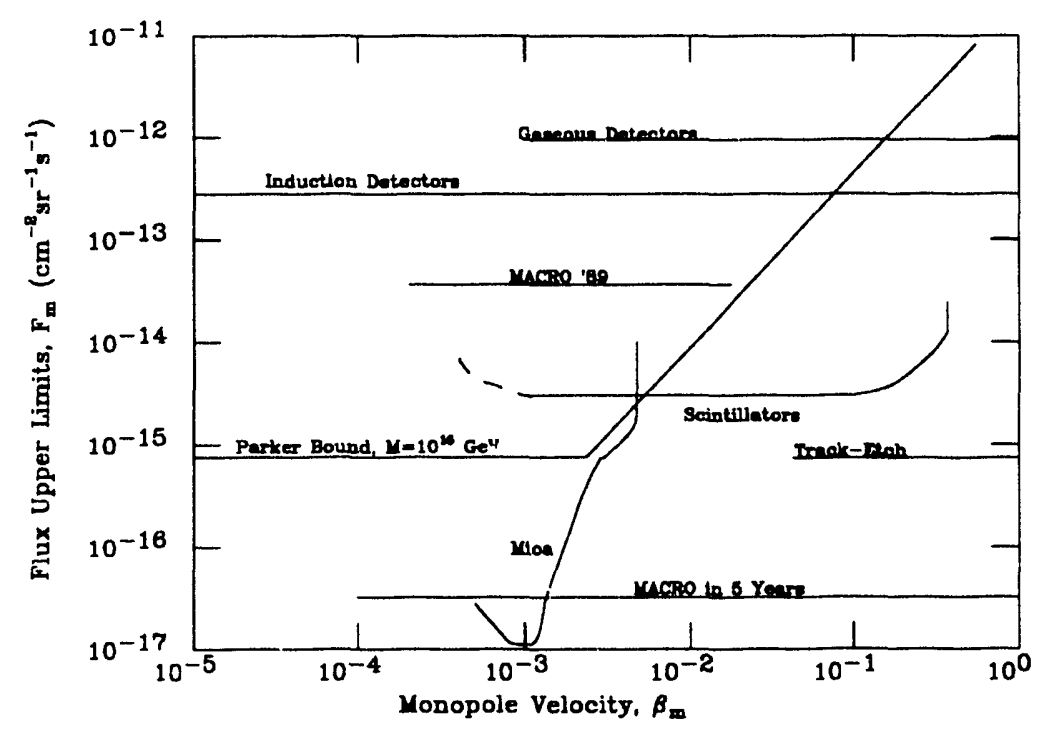

Figure 1: Astrophysical and experimental bounds (1989) on the flux of GUT monopoles.

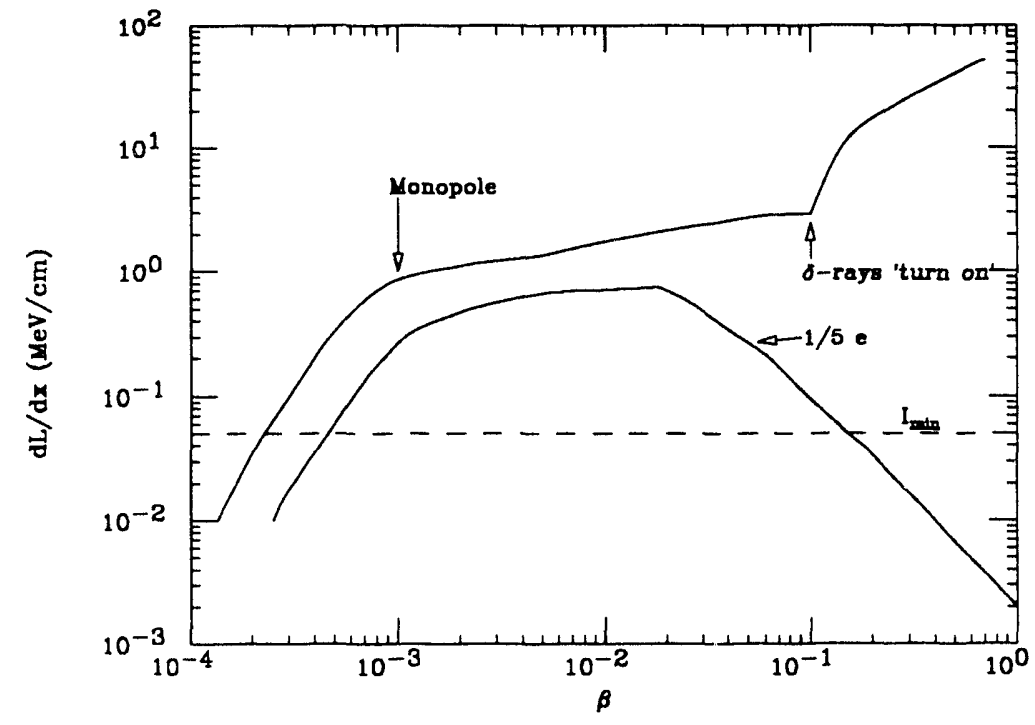

Figure 2: Expected scintillation yield, $d L / d x$, for a magnetic monopole and for a charge $1 / 5 \epsilon$ particle compared to that of a minimum ionizing particle, $I_{\min }$. The efficiency of the scintillator for converting ionization energy to light is $2.5 \%$. A minımum ionizing particle whose ionization rate is $2 \mathrm{MeV} / \mathrm{cm}$ therefore has a scintillation yield of $.05 \mathrm{MeV} / \mathrm{cm}$. 


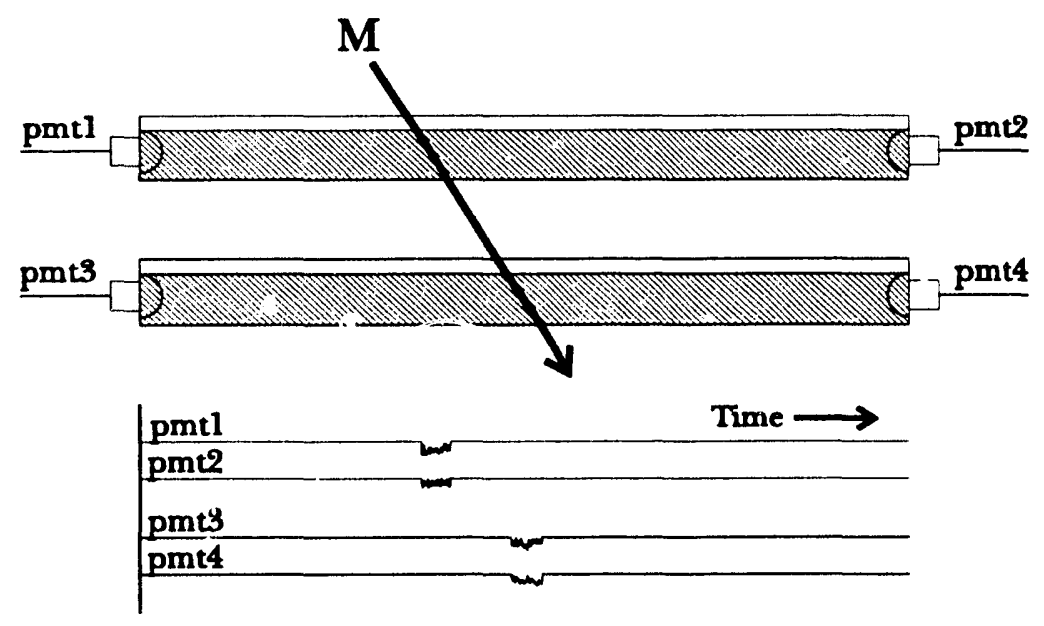

Figure 3 : Experimental signature for a monopole passing through two layers of MACRO. The pulse widths are from the finite module transit time of the monopole.

It should be noted that while the time and $\mathrm{pu}_{\mathrm{i}}$ ie-shape signatures of monopoles distinguish them from cosmic rays and other backgrounds, there are many species of proposed massive particles that could produce a similar signature. This possibility is less a problem than an opportunity, since the detection of any such massive particle would signal new physics at very high energy scales.

\subsubsection{Trigger design}

Unlike triggers for 'slow' $\mathrm{m}$. . opoles $\left(\beta<10^{-3}\right)$, it is not necessary to have very low thresholds to be sensitive to fast monopoles. At the same time, the fast monopoles transit a scintill ition counter quickly enough that the pulse shape from the PMT's does not give a very good means of distinguishing fast monopole candidates from cosmic ray muons at the trigger level (see Fig. 4). Signals from muons or radioactive decays very near a PMT that saturate the P.IT and give 'square' pulses can mimic the pulse shape of a fast monopole. It is unlikely, however, that background can give a particle track with 3 significantly less than one. 


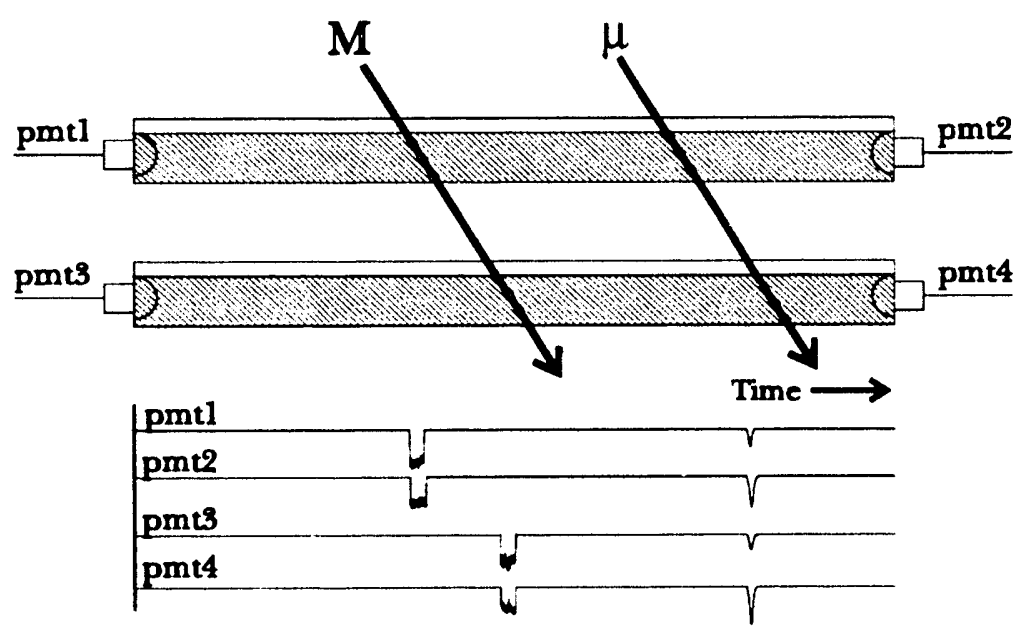

Figure 4: Comparison of signatures in MACRO for cosmic-ray muons and fast monopoles.

The main difficulty for a fast monopole trigger is the copious flux of cosmic ray muons with $\beta=1$. A combination of pulse amplitude and timing discrimination is required to reject muons with the necessary efficiency. This rejection cannot be done on a 'single counter' basis, however, since one of the primary signatures for a muon is its time-of-flight between MACRO counters. Instead. we will use a delayed coincidence between different planes of scintillation counters to reject prompt $(\beta=1)$ background. Some geometric inefficiency thereby will necessarily be introduced due to cracks and dead spaces in MACRO. and because of the need to limit the number of counters that can participate in the trigger.

For the scheme described below with an isotropic flux of fast monopoles, we have calculated that there is a $\approx 15 \%$ geometric inefficiency, mostly due to "corner clippers' that have very short transit times through MACRO. A further stage of data analysis of muon events may be able to recover some of the efficiency lost to such geometric effects.

In five vears of running with six supermodules, the number of cosmic 
ray muons expected (scaling irom the Spring ' 89 run data) is about $3 \times 10^{7}$. During that same time, one might expect a few fast monopole candidates.

Because of the very low flux limits that MACRO will be able to probe, it is possible that there is indeed a detectable monopole flux. This possibility mandates that monopole triggers cause an extensive readout of the detector. since each trigger could be a long-awaited monopole going through the detector.

The extensive readout makes it very expensive in terms of deadtime and data rate to produce false monopole triggers, and puts a premium on the rejection capabilities of the monopole trigger logic. Not only must the triggers be efficient, but they need to be noise free.

The trigger design which will be implemented for MACRO consists of three 'subtriggers', to cover the area of interest in the $d L / d x$ vs $\beta$ plane while efficiently rejecting cosmic-ray muons. Such a design will give MACRO full sensitivity to fast monopoles as well as sensitivity to massive fractionally charged particles (such as the $1 / 5 e$ particles suggested in some string theories [20]), together with an active muon veto that can be used as a prompt muon trigger in global muon trigger logic.

The $\mu$ trigger is a fast coincidence of scintillator planes, suitable for use as a global muon trigger and as a muon veto for monopole triggers. The FASTM trigger is a delayed coincidence of scintillator planes, with a veto from the $\mu$ trigger to exclude muons. The highly-ionizing particle trigger (HIPT) is also a delayed coincidence of scintillator planes, with a higher threshold.

The trigger logic (see Fig. 5) uses signals from fanned-in sets of 8 scintillation counters. The combined signals are discriminated, and a coincidence of two ends of the set of scintillation counters is required within a short time window together with a coincidence of two 'planes' of MACRO within a longer time window.

We have recently developed and put into limited production an 8:1 linear fanin with coaxial ribbon cable input having normal and fast-integrated signal outputs. Discriminating on a signal that is integrated with a timeconstant of $\sim 50 \mathrm{~ns}$ gives a slower drop-off of discriminator sensitivity as a function of monopole velocity, which is desirable for fast monopole detection.

Fig. 6 shows the regions in $\beta$ and $d L / d x$ which are covered by the FASTM, HIPT and $\mu$ veto triggers. The FASTM trigger is vetoed by the $\mu$ trigger to prevent muons from swamping the fast monopole triggers. HIPT trigger levels are set at $\sim 10 \times I_{\min }$ which should be sensitive to very fast monopoles, 


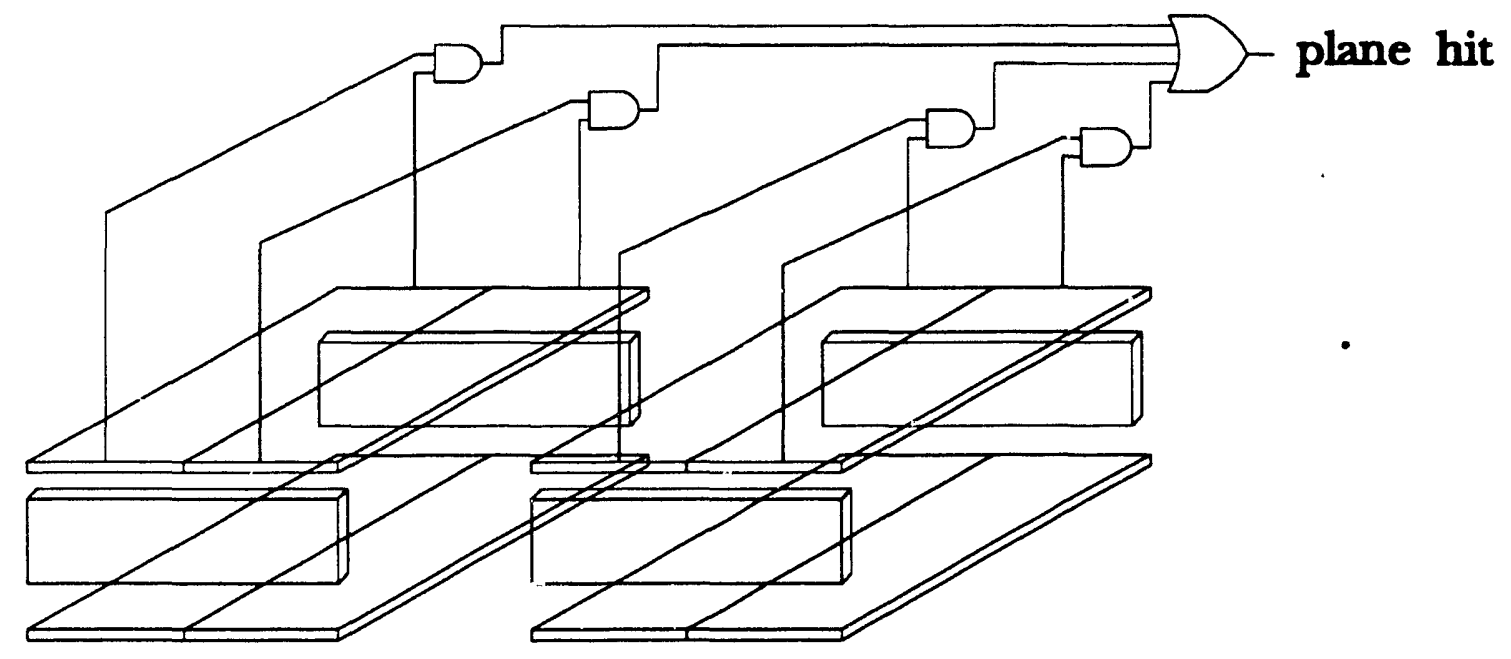

Supermodule 1

Supermodule 2

Figure 5: The fast monopole trigger logic uses an end-to-end coincidence of signals from a set of 8 scintillation counters, and combines these coincidence signals to make a 'plane hit' signal. Two or more planes being hit within a coincidence interval produces a trigger.

yet have a low false trigger rate from muon events. Saturation effects in the PMT's can make for difficulties in implementing a high-threshold trigger; the saturation characteristics of the PMT's is a subject of active study at this time.

These triggers will be combined for adjacent overlapping pairs of supermodules to provide more complete angular coverage for fast monopoles. If singles rates allow. this combination of triggers can be extended to include more supermodules in an individual triggering group. 


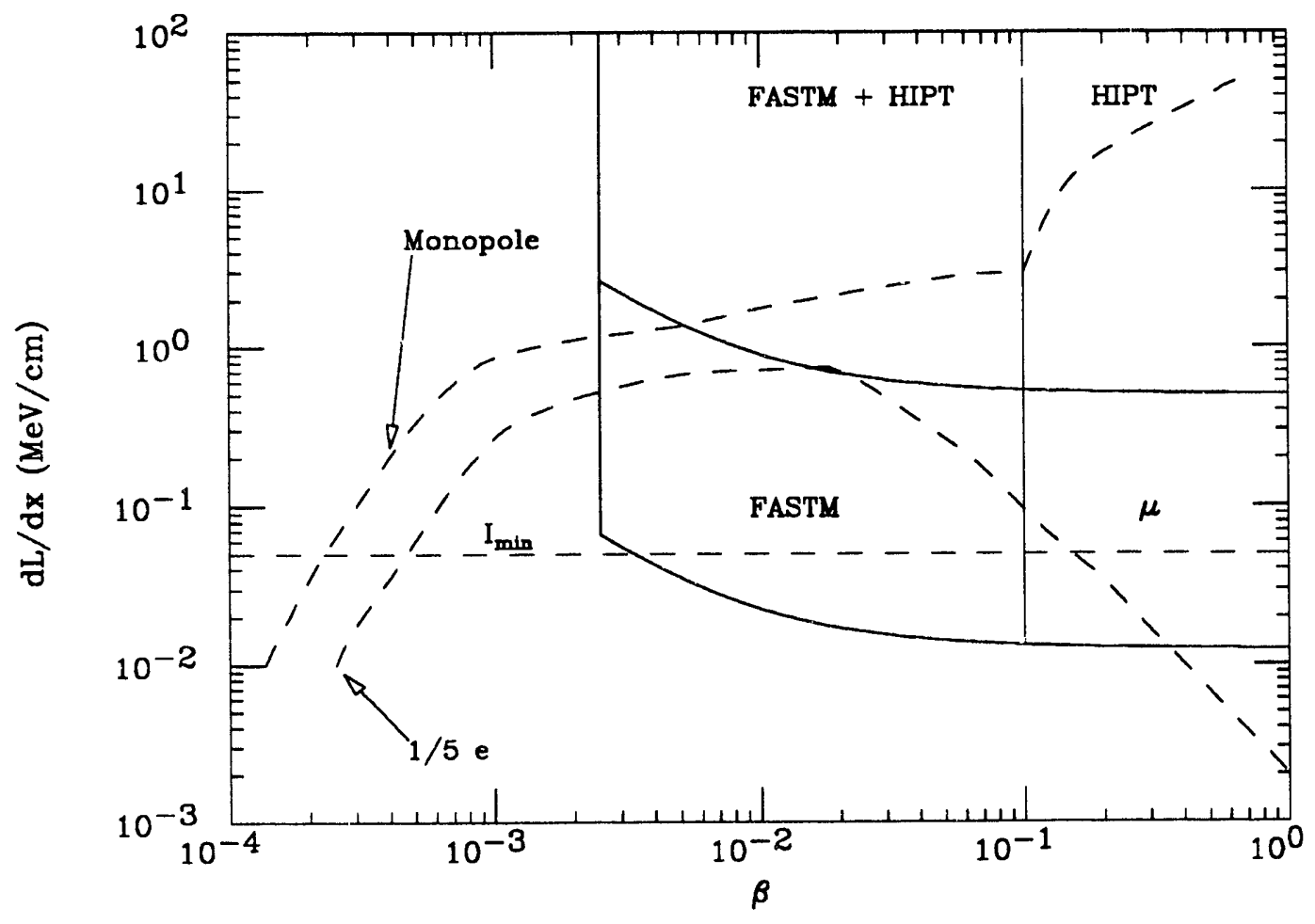

Figure 6: Coverage of the FASTM, Highly-Ionizing Particle (HIPT) and $\mu$ veto triggers in $d L / d x$ vs 3 . The triggers are effective for GUT monopoles with $\beta>2.5 \times 10^{-3}$ and for $1 / 5$ e particles with $2.5 \times 10^{-3}<\beta<0.1$. For explanation of $d L / d x$ see Fig. 2 caption. 


\subsubsection{Implementation}

Overview Achieving the capability required of the fast monopole trigger within a limited budget has required us to design some custom electronics. The fast monopole trigger operates with highly fanned-in signals to reduce the channel count of trigger electronics. Even so, the number of channels needed for MACRO is quite large.

Signals from the PMT's are fanned out to a number of trigger and acquisition systems; the signals for the fast monopole trigger are sent through a splitter printed circuit board (PCB) to obtain the correct combination of signals on each cable, then linearly added to make a 'patch end' signal for 8 scintillator tanks.

The patch ends are discriminated at $\approx 100 \mathrm{mV}$ in a commercial programmable high-density discriminator; an end-to-end fast coincidence is then required of the discriminated signals to make a 'patch hit'. Up to four adjacent patches on the same plane of MACRO are then ORed to make a 'plane hit', and a 2 fold coinc: dence of plane hits is used to trigger the experiment.

The custom components of this system are the splitters, fanins, and plane logic for patch-end AND/ORing.

Linear fanins The first challenge for a linear fanin was in achieving an 8-to-1 analog fanin of PMT signals with sufficient dynamic range for fast monopole signals. Commercial fanins have strictly limited range (typically $1 \mathrm{~V})$. and the most attractive possibility for a commercial $8: 1$ fanin is no longer in production.

As a result, it was necessary for us to design and build a fast analog 8:1 fanin. The fanin was developed and tested in prototype form in the summer of 1990: a CAD (computer automated design) program was used to generate a custom PCB layout for commercial board production. The fanin was sent out for commercial production in early 1991, and we obtained the final PCB's in April.

A sufficient number of fanin modules have since been assembled and tested to implement a fast monopole trigger using two supermodules (excluding the attico). Production of the full number of PCB's needed for all of MACRO will proceed after a trigger test conducted with supermodules 2 and 3 .

Fig. $T$ shows the current linear fanin design. Major criteria for this fanin are linearity for small pulses and a large dynamic range, since it is expected 
that fast monopoles will produce very large PMT pulses. The fanin schematic shown has a $6 \mathrm{~V}$ input dynamic range, a gain of about 0.66 in its linear range, and a PSRR (power supply rejection ratio) of less than $0.1 \%$.

Both the linearity and the dynamic range of the current fanin design are slightly less than achieved with early prototypes because of the need for added stability under a variety of load conditions. Fig. 8 shows the output voltage as a function of input voltage on one channel for one of the production fanins. The saturation shown in the figure is occurring on the input circuitry for the fanin; the output circuitry saturates at a higher level, which allows summation of saturated signals. Because the signals out of the fanin are being discriminated rather than measured for acquisition, the nonlinearity for large input signals is not expected to be problem.

The channel-to-channel variation of the fanin response functions is quite small, particularly in the linear response region. Fig. 9 shows response functions for all eight channels of a fanin. The variation between different fanin units is also quite small, allowing global thresholds to be used on fanned in signals without the need for individual adjustments.

The fanin is designed to be appropriate for implementation on a thick-film hybrid or on a bipolar chip; however, a discrete component implementation is used for MACRO because of the necessity for timely deployment. If further uses of this fanin are found, a more elegant packaging of the circuit will be investigated.

Signal Splitter In addition, a 'signal splitter' $\mathrm{PCB}$ was designed and produced in quantity so that the signals available from the MACRO PMT fanouts could be recombined into the groups needed by the fanins.

Our cost studies show that close to half of the fanin cost is a 'per module' cost. regardless of how many functional units are contais ed in a module. By combining the signals appropriately, we can use a $50 \%$ higher circuit density on the fanin PCB's. and decrease the overall cost of the trigger.

The signal splitters take signals from two input cables in the form $1 R$, 1L. 2R. 2L. ... and route them to two output cables in the forms $1 \mathrm{R}, 2 \mathrm{R}$, $\ldots$ and $1 \mathrm{~L}$. 2L.... suitable for fanning in to make plane-end sums.

The trace widths required to maintain a constant characteristic impedance on the splitter PCB mandated the use of $93 \Omega$ coax ribbon cable for the inputs to the fanin. which also has the advantage of reducing the current load 


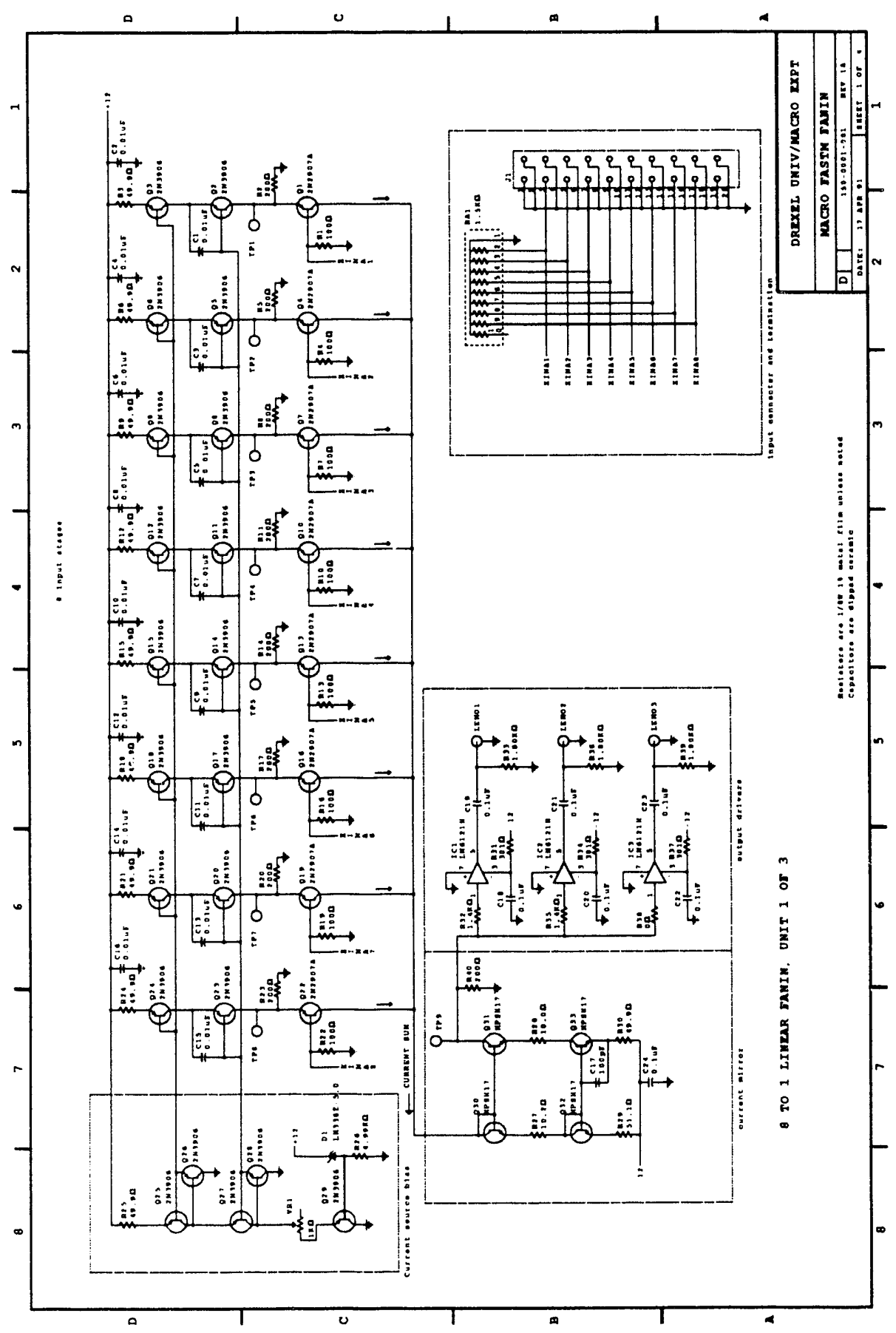

Figure 7 : Schematic for the fast linear 8:1 fanin. Current from each input stage is summed at the input of a current-mirror. Two of the fanin outputs are integrated with time constants of $\approx 50 \mathrm{~ns}$. 


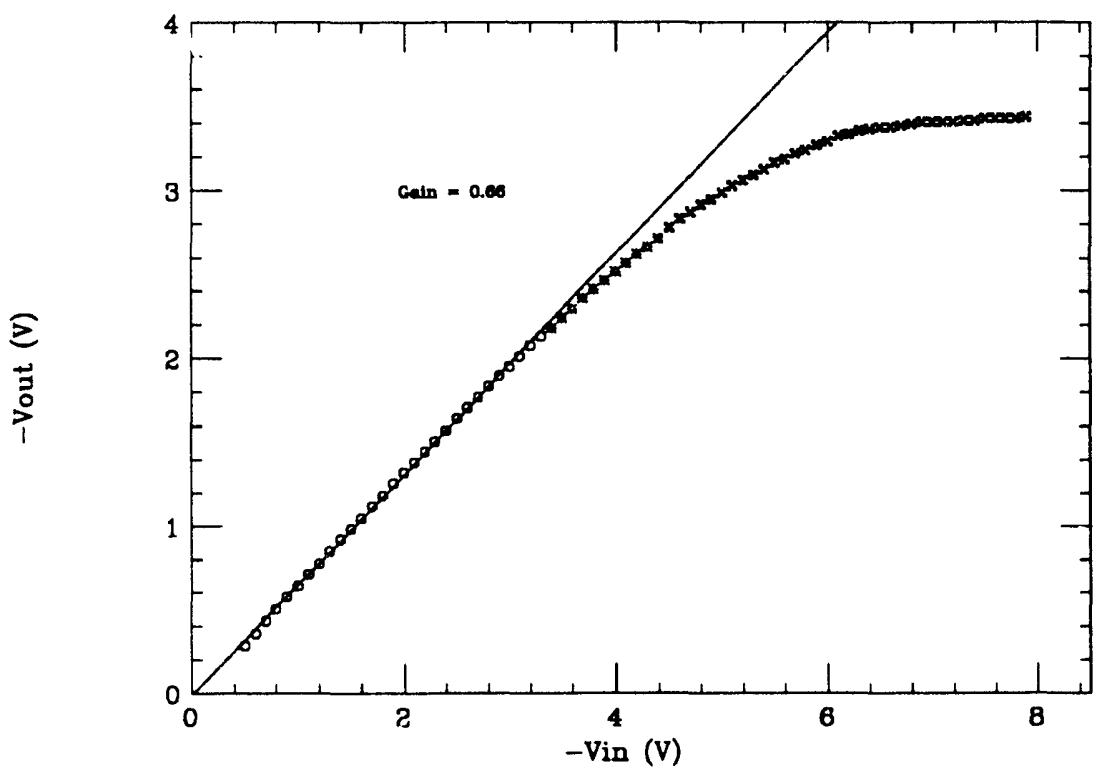

Figure 8: Response function of production fanin, showing linearity up to $\approx 3 \mathrm{~V}$, with saturation at an input of $\approx 6 \mathrm{~V}$.

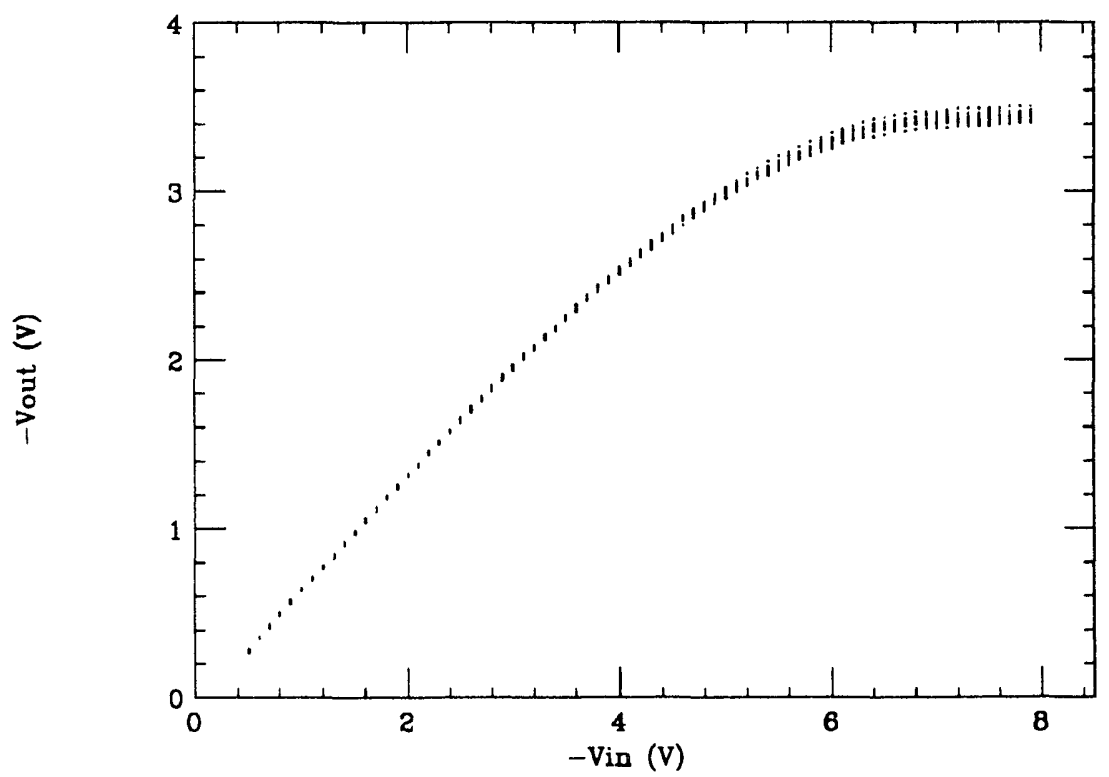

Figure 9: Response function of 8 inputs of fanin plotted together. Similar plots of different fanin units are nearly identical to this one. 
on the fanouts driving the ribbon cables. The fanin design allows a simple change of input characteristic impedance by changing a single resistor array on each input connector.

Six signal splitter PCB's are bolted to a rack-mount panel and provide signals for four 8:1 fanin modules.

Plane Logic Module A prototype 'plane logic' module was designed and constructed in May 1991 for use on MACRO this summer. This module makes the fast coincidences of signals from opposite ends of a group of tanks, and also combines signals from the same plane of MACRO.

Fig. 10 shows the coincidence logic used in the plane logic module; the schematic is part of the preliminary design for the production model. We expect the final version of the plane logic module to be ready in the Fall of 1991 and have added capabilities for trigger pattern storage and singles rate monitoring. Because of the small number of plane logic modules needed for MACRO (approximately 10) the PCB's for this module will probably be made in-house. with commercially photoplotted artwork.

Cables A significant expense in the fast monopole trigger is the large number of ribbon coax cables used for primary signal inputs. After consultation with cable manufacturers and companies specializing in cable assembly, we found that it would be most cost-effective to purchase the cable making equipment and to assemble the cables on-site to the necessary lengths. We expect the cable-making facilities to be generally useful in MACRO and other future experiments using many channels of analog information.

Calibration The fast monopole trigger scheme has been developed in parallel with a scheme for calibration and monitoring of the detector and the trigger system. Since the flux of monopoles is extremely low we lack a test beam to see that our triggers are efficient. Our calibration and monitoring scheme uses a computer program (shown as a 'thought balloon' in Fig. 11) which generates an isotropic flux of fast monopoles through MAC'RO. The pathlengths. $d L / d x$, and scintillator response curves are factored in to give the pulse height. width. and relative delay between P.MT pulses in two scintillator counters (or two P.MT's in bench-testing). The P.MT response in photoelectrons is calibrated for given widths and pulse heights of LED driv- 


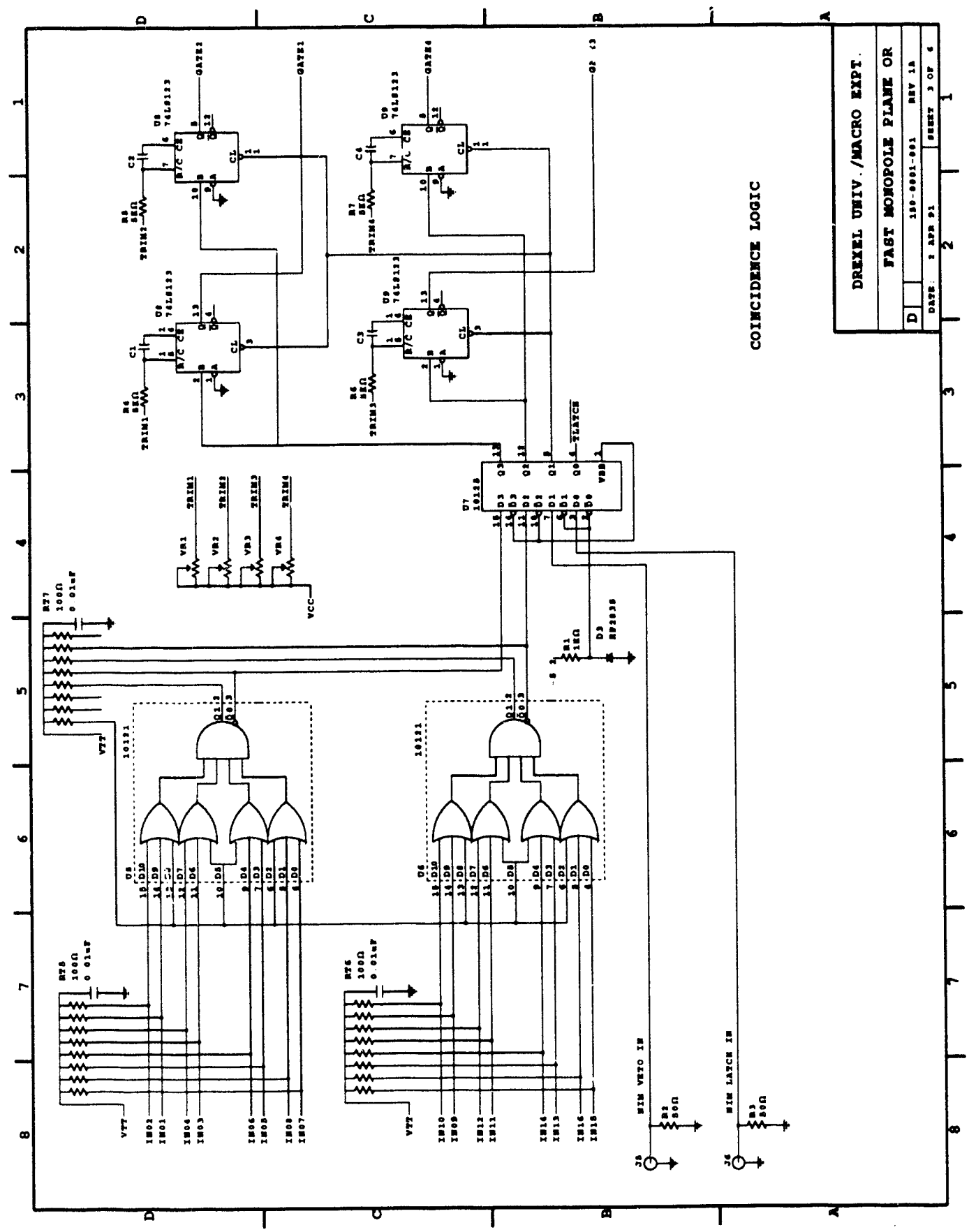

Figure 10: Partial schematic for the plane logic module. The patch-end input signals are combined in an end-to-end AND, with an OR between patches on the same plane. This function is contained in a single 10121 ECL chip using negative logic. 
ing pulses, and the intelligent pulse generator is given a set of instructions which result in a pair of pulses being sent out to the LED's.

Our calibration scheme thus produces PMT pulses which simulate a monopole's passage through MACRO, and can test the fast (and slow) monopole trigger efficiency for isotropic monopoles as a function of $\beta$, as well as testing for effects such as saturation of the PMT's or electronics with very large signal pulses.

This calibration system is being implemented jointly with the MACRO group at Boston University, which has responsibility for the development of relay modules for switching pulses to the appropriate MACRO scintillator tanks. 

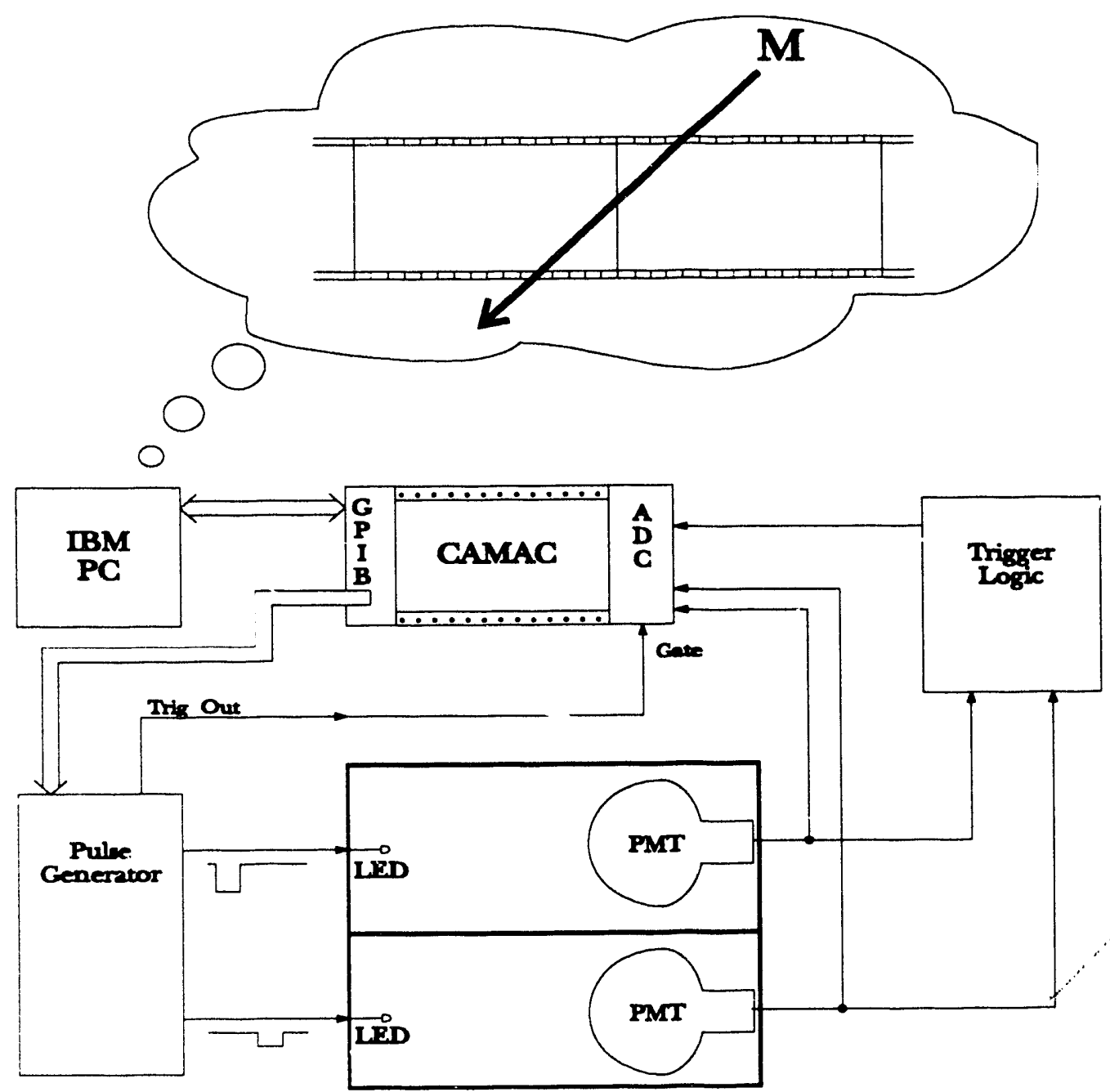

Figure 11: Calibration and testing scheme for fast monopole triggers. A r.ogram on the IB.M-PC' 'generates' an isotropic flux of fast monopoles, and fires calibrated LED pulses to simulate the monopoles in MACRO. This system can also be used with PMT's in dark boxes (as shown) to test the trigger logic at Drexel. 


\subsection{MACRO data analysis}

The calibration procedures for MACRO are currently under revision, with the primary goal being to improve the quality and completeness of the calibrations. This revision will require a closer connection between the calibration requirements for analysis and the calibration process.

We intend to automate the calibration as part of the online acquisition system (currently it is a standalone system), and to upgrade the calibration hardware to allow for more precise, higher statistics LED calibration runs. These improvements should benefit the monopole and neutrino burst triggers, since each of them depends critically on the setting of low-energy detection thresholds.

As described above (see Section 2.2.4), we are developing calibration and testing procedures for the monopole electronics, and expect the first part of the system described above will be installed on MACRO this summer.

Our data analysis program is being strengthened by the addition of John Petrakis, who is devoting $25 \%$ of his time to MACRO data analysis. Dr. Petrakis is a postdoc at the Bartol Institute, and brings with him a wealth of experience in MACRO data analysis. The first goal of our analysis program is to search for delayed muons in multi-muon events.

In addition. one of our graduate students, Octavia Blount, has been working on time distributions of single muon events as a signature of cosmic ray anisotropies.

The available MACRO data has increased greatly over the past year; the Spring ' 89 run produced over 200,000 muon events and the Fall '90 run another 300,000. In May 1990, MACRO started taking muon data with two supermodules. and by February 1991 had accumulated another 1,100,000 muons.

The continuing strain of data tape copying, distribution and storage has pushed the collaboration to the use of compact $8 \mathrm{~mm}$ digital cartridge tapes as a medium. These tapes are now used for data distribution and much of the analysis of MACRO data.

In early 1991, we purchased an Exabyte $8 \mathrm{~mm}$ tape drive in order to analyze the current data tapes, and also upgraded the CPU of our MicroVAX to increase the CPL power available for analysis. The upgrade achieved a raw CPL performance increase of about 3.5 at modest cost. 


\subsection{Shifts and on-site work}

Construction and operation of the MACRO experiment requires constant availability of significant manpower. To share the load equitably, a system of rotating shifts requiring each collaborator to work at the experimental site for two weeks once or twice per year has been set up. In addition, Prof. Lane and Michael Mittelbrunn are spending additional time at Gran Sasso for the installation of fast monopole trigger electronics. We expect that $\mathrm{Mr}$. Mittelbrunn will also be contributing MACRO shifts in the coming months.

Supermodules 2 through 4 are being brought on-line, and many problems of nolse and triggering are being encountered. Prof. Lane solved many such problems during 1989-90 when he was the on-site U.S. MACRO supervisor. He is aiso contributing to the preparation of the new supermodules for data taking. 


\section{Search for neutrino oscillations}

\subsection{Introduction}

One of the central issues in particle physics, astrophysics and cosmology is the possibility of the existence of a nonzero neutrino rest mass. In the standard $S L_{2} \times U_{1}$ electroweak model, the neutrinos are massless. However, the minimal $S U_{2} \times U_{1}$ theory is generally believed to be unsatisfactory and therefore unlikely to offer a complete theory of the electroweak interaction. Many extensions of the standard model involving new Higgs representations or new $S L_{2}$-singlet neutral fermions (for example, most grand unified theories other than $\left.S C_{5}\right)$ are consistent with non-zero neutrino rest masses. There is therefore good motivation for experimental searches for a finite neutrino rest mass.

\subsection{Experimental searches for neutrino mass}

From the experimental point of view, there are a number of approaches to detection of a finite neutrino rest mass. Precision measurement of low energy beta decay spectra in the vicinity of the end point (i.e. zero neutrino kinetic energy) is perhaps the most direct approach. Currently, limits provided by this technique are in the vicinity of $m_{\bar{\nu}_{\varepsilon}} \sim 10 \mathrm{eV}$. In addition, there are indications at present of the possible existence of a small admixture of a 17Kel neutrino $\left(\nu_{+}\right.$?) in the electron neutrino. but much controversy exists.

Other methods sensitive to neutrino mass are observation of the time structure of neutrino pulses from collapsing stars, and searches for neutrino matter and vacuum oscillations. The oscillation searches have the very great advantage of exquisite sensitivity to the mass of the oscillating particles. For example. the observed strangeness oscillations in the $K^{0} \cdot \bar{K}^{0}$ system reveal an underlying mass difference of $\Delta m=3.5 \times 10^{-6} \mathrm{eV}$ !

\subsection{Nev'rino vacuum flavor oscillations}

Only a single assumption. that of nonconservation of separate lepton number, is required to seek proof of finite neutrino mass by searching for neutrino racuum oscillations. Since there is no compelling reason for believing in the separate and absolute conservation of $L_{\epsilon} . L_{\mu}$ and $L_{\tau}$. the existence of mass 
differences among the neutrino mass eigenstates $\left(\nu_{1}, \nu_{2}, \nu_{3}\right)$ would lead to their propagation with slightly different frequencies.

Hence, an initially pure (i.e. $\therefore$ perfect eigenstate of the weak interaction) beam of $\bar{\nu}_{t}$. which at the same time would be a coherent superposition of neutrino mass eigenstates, would develop at subsequent times a $\bar{\nu}_{\mu}$ component, while the $\bar{\nu}_{\epsilon}$ content of the propagating beam would undergo a corresponding decrease. An experimental measurement of the decrease of the $\bar{\nu}_{\epsilon}$ flux would therefore detect the presence of the neutrino oscillation and hence reveal the underlying finite neutrino rest mass. This type of measurement is known as a neutrino oscillation disappearance experiment and has the great advantage of being sensitive to extremely small values of neutrino mass differences. In addition, disappearance experiments are sensitive to several reaction channels simultaneously. For example, $\bar{\nu}_{\epsilon}$ might oscillate into $\bar{\nu}_{\mu}$ or $\bar{\nu}_{\tau}$, or even into $\nu_{\epsilon}$.

Alt ernative methods of testing for neutrino vacuum oscillations by searching for the appearance of an otherwise absent neutrino flavor are also of interest and frequently offer the advantage of being sensitive to neutrino oscillations of rather small amplitude (i.e. having a small mixing angle, $\theta$ ). This type of experiment usually explores only a single reaction channel, which, depending on circumstances. could be either an advantage or a disadvantage.

The possibility of neutrino vacuum flavor oscillations was first envisaged by Maki (1962) and by Pontecorvo (1968).

\subsection{Reactor neutrino oscillation experiments}

The sensitivity of a neutino oscillation search by means of an appearance channel is governed in a two-flavor scenario by the equation for the probabilit $y P\left(\nu_{f} \rightarrow \nu_{x}\right)$ that a neutrino of the first flavor will oscillate into a neutrino of the second flavor. e.g.

$$
P\left(\nu_{\epsilon} \rightarrow \nu_{x}\right)=\sin ^{2} 2 \theta \sin ^{2}\left(\frac{1.27 \Delta m^{2}\left[\mathrm{eV}^{2}\right] L[\mathrm{~m}]}{E_{\nu}[\mathrm{MeV}]}\right),
$$

where $\theta$ is the mixing angle, $\Delta m$ is the mass difference between the neutrino mass eigenstates. $L$ is the source-detector distance, and $E_{\nu}$ is the neutrino energy: 
For a disappearance experiment, the relevant quantity is the probability $P\left(\nu_{\epsilon} \rightarrow \nu_{\epsilon}\right)=1-P\left(\nu_{\epsilon} \rightarrow \nu_{x}\right)$. In either case. the experimental sensitivity is determined by the ratio $L / E_{\nu}$. It is therefore important to use the largest value of $L$ consistent with adequate statistical information (hence a desire for long baseline experiments). At the same time, working at the lowest practical neutrino energy is also important.

Nuclear power reactors provide intense, rather well understood sources of low energy $\bar{\nu}_{\epsilon}$ 's. Over the years therefore, a number of neutrino oscillation searches have been performed at various reactors. Probably the most significant of these experiments is that performed at the Gösgen nuclear power reactor in Switzerland [21] by a group under the leadership of F. Boehm.

The Gösgen experiment measured the $\bar{\nu}_{\epsilon}$ spectrum at distances up to 64.7 meters from the reactor by using the inverse beta decay reaction $\bar{\nu}_{\epsilon}+\mathrm{H} \rightarrow \epsilon^{+}+n$. The reactor had a thermal power of $2800 \mathrm{MW}$. while the detector provided a target mass of $320 \mathrm{~kg}$ and yielded a neutron detection efficiency of $21.7 \%$.

The results of neutrino oscillation experiments are often displayed on socalled exclusion plots, such ai that shown in Fig. 12. In this plot, the area above and to the right of the curve labelled 'Gösgen' is the region of the $\Delta m^{2}$ is $\sin ^{2} 2 \theta$ phase space where neutrino oscillations would have been detected by this experiment had they been present. The minimum detectable $\Delta m^{2}$ for maximal mixing was $1.9 \times 10^{-2} \mathrm{eV}^{2}$. 


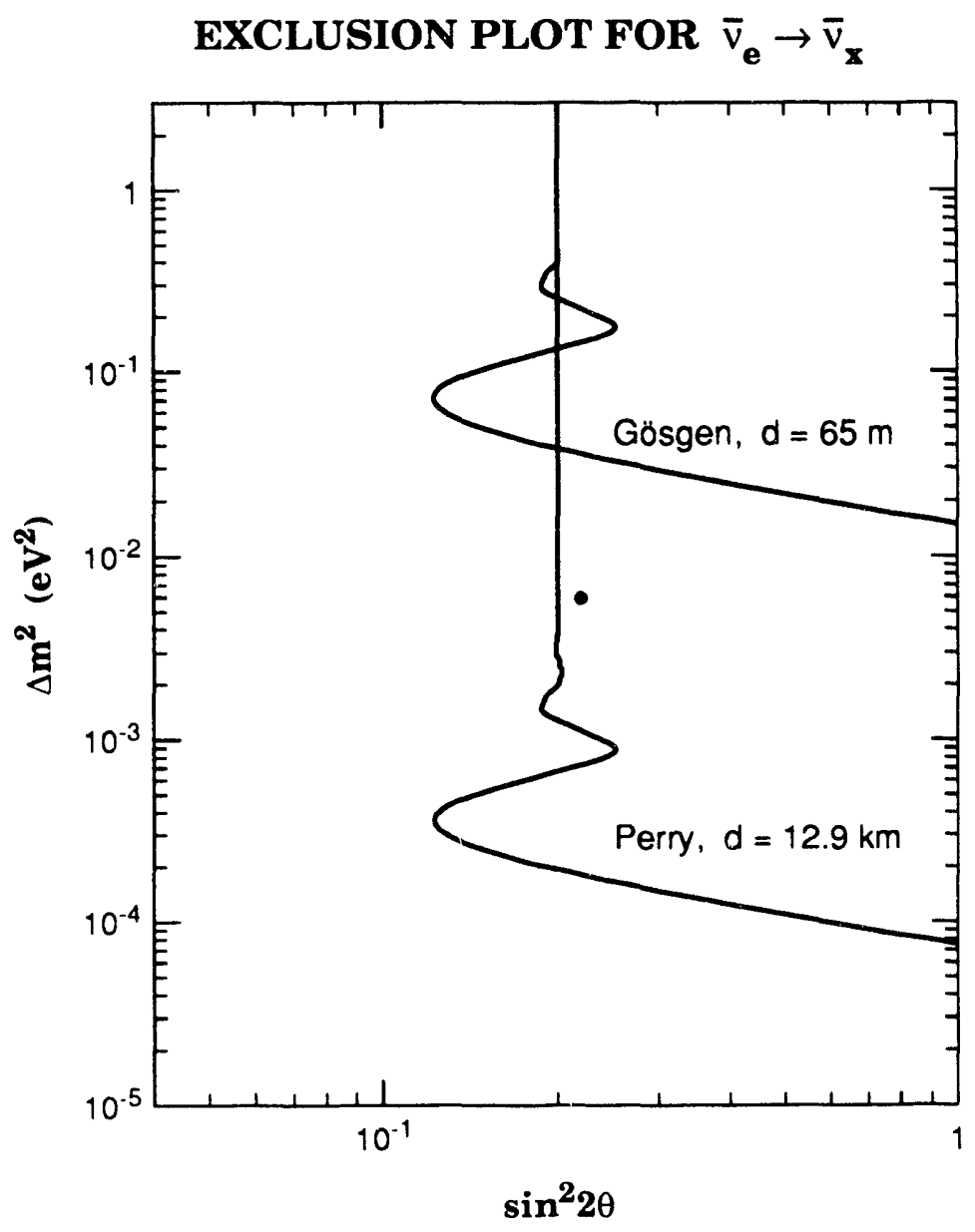

Figure 12: Limits on neutrino vacuum oscillations obtained in the Gösgen experiment and to be reached in the proposed Perry experiment. 


\section{The Perry experiment}

\subsection{Overview}

Together with the present members of the IMB collaboration, we have submitted to the Department of Energy a letter of intent to perform a new highly sensitive neutrino oscillation experiment taking advantage of existing equipment and experience at the IMB Fairport (Ohio) underground facility and utilizing the Perry Nuclear Power Reactor of the Cleveland Electric Illuminating Co., which is optimally situated at a distance of $12.9 \mathrm{~km}$ from the underground laboratory.

The limits on neutrino vacuum oscillations expected from the experiment are shown in Fig. 12 by the curve labelled 'Perry'. For maximal mixing, the experiment will detect oscillations for values of $\Delta m^{2}>8 \times 10^{-5} \mathrm{eV}^{2}$, covering the two-order-of-magnitude gap between the currently available limits and those potentially obtainable from solar neutrino experiments.

The concept of the experiment, as shown in Fig. 13, is to suspend a $12 \mathrm{~m}$-diameter transparent spherical acrylic container in the center of the $23 \mathrm{~m} \times 17 \mathrm{~m} \times 18 \mathrm{~m}$ IMB swimming pool. About 900 tons of a highly transparent chlorinated liquid scintillator (under development at Drexel, see Section 4.4. page 34) with a density slightly greater than that of water (to reduce stresses on the acrylic vessel) will permit efficient and nearly background free detection of the positrons and neutrons resulting from the inverse beta decay reaction of the $\bar{\nu}_{f}$ 's on the hydrogen atoms of the scintillator. Scintillation photons from particle interactions in the vessel will be collected by the existing 2048 eight-inch photomultipliers and processed by zero deadtime multi-hit adc/tdc's.

The 8000 tons of ultra-pure water in the IMB facility will serve both as passive shielding against ambient radioactivity and as active shielding against cosmic ray muon-induced backgrounds, which already will have been strongly suppressed by the 600 meters of rock overburden.

Neutrinos above the threshold energy of $1.8 \mathrm{MeV}$ will be detected by the reaction $\bar{\nu}_{\epsilon}+\mathrm{H} \rightarrow \epsilon^{+}+n$. The observable energy from this reaction will equal the positron kinetic energy augmented by the $1.022 \mathrm{MeV}$ resulting from essentially calorimetric detection of the positron annihilation gamma rays. Following thermalization of the emitted neutron, an additional $8.6 \mathrm{MeV}$ will be detected as a result of capture of the thermalized neutron by a ${ }^{35} \mathrm{Cl} \mathrm{nu}$ - 


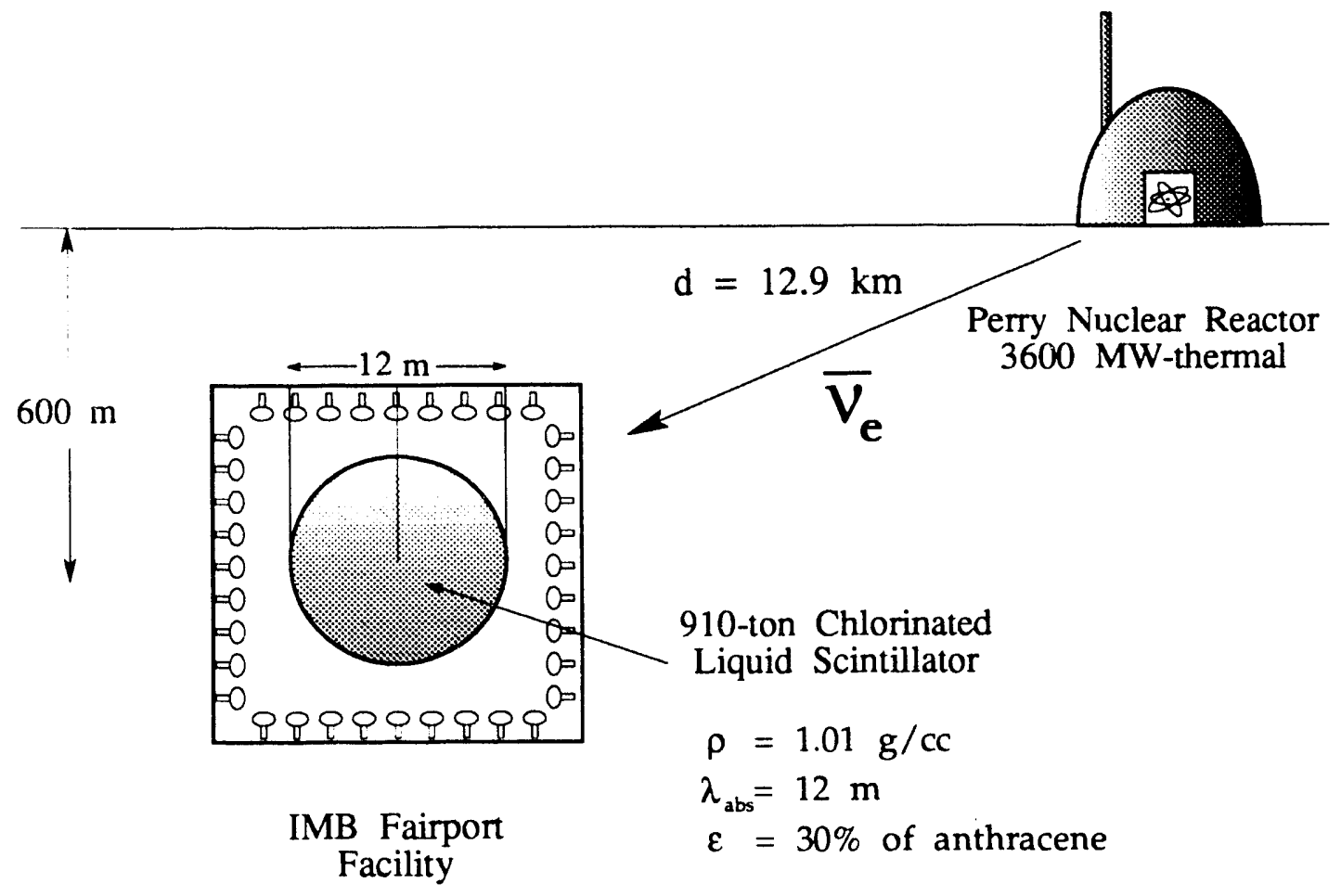

Figure 13: Concept of the proposed long baseline neutrino oscillation experiment using the Perry reactor and the underground IMB Fairport Facility.

cleus (1otal time required $\sim 34 \mu s$ ). Thus, a readily recognizable delayed coincidence pulse pair will signal the neutrino interaction. The $8.6 \mathrm{MeV}$ neutron capture event will be well separated from the beta and gamma radiation accompanying decay of members of the ubiquitous uranium and thorium decay chains and from the beta decay of ${ }^{40} \mathrm{~K}$. Further significant rejection of the accidental coincidence background will be possible by reconstructing the positron and neutron capture vertices and demanding a spatial separation not to exceed 2 meters. 


\subsection{Event rate}

The neutrino flux for the Perry experiment is reduced with respect to that in the Gösgen experiment by a factor of $(12900 / 64.9)^{2}(2.8 / 3.6)$ or about 30,000 . The event rate is kept reasonable by scaling up the target mass by a factor of $(910 / .32)=2800$ and by increasing the efficiency of the detector from $21.7 \%$ to $\sim 80 \%$. Together, the mass and efficiency increases allow for a factor of 10,000 improvement, so that the expected event rate remains satisfactory.

We calculate the expected antineutrino event rate in the Perry experiment to be about $12 \mathrm{~d}^{-1}$ using the assumptions shown in Table 1 . For a 2-year run we thus anticipate nearly 9000 events, which would be adequate since systematic effects will, in any case, limit the measurement accuracy to about $5 \%$.

\begin{tabular}{|l|l|}
\hline Reactor power & $3.60 \mathrm{GW}-$ thermal \\
Energy release per fission & $203 \mathrm{MeV}$ \\
$\bar{\nu}_{\epsilon}-\mathrm{H}$ cross section & $6.3 \times 10^{-19} \mathrm{~b} /$ fission \\
Fission rate & $1.11 \times 10^{20}$ fissions $/ \mathrm{s}$ \\
Reactor distance & $12.9 \mathrm{~km}$ \\
Target H density & $6.3 \times 10^{22}$ atoms $/ \mathrm{cm}^{3}$ \\
Detector radius & $6 \mathrm{~m}$ \\
Detector volume & $905 \mathrm{~m}^{3}$ \\
Detector efficiency & $75 \%$ \\
Event rate & 12.3 events $/ \mathrm{d}$ \\
\hline
\end{tabular}

Table 1: Inverse beta decay event rate in the Perry experiment. 


\subsection{Backgrounds}

As in many neutrino experiments, a major task in the Perry experiment is to reduce the background to the low levels necessary for clean observation of the signal. Fortunarely. the delayed coincidence signature for the antineutrino inverse beta decay events, together with a tight spatial coincidence requirement. should obviate the need for the heroic effort required in such singles counting experiments as SNO.

The backgrounds are of three principal types:

Entering radioactivity Low energy neutrons and gamma rays originating in the rock walls of the facility should be reduced to very low levels by the three meters of water shielding. Fission neutrons are attenuated by a factor of $10^{4}$ by each meter of water: $2-\mathrm{MeV}$ gamma rays are reduced 170 -fold per meter of water. while at $10 \mathrm{MeV}$ the reduction factor is about 10 per meter. Entering radioactivity should therefore be no problem. We plan to make in situ measurements of the singles count rates to verify this situation (see Section 4.6).

Cosmic ray backgrounds Through-going cosmic ray muon-induced backgrounds should be manageable because, at the $1570 \mathrm{MWE}$-depth of the IMB site. the measured muon rate is about $3 \mathrm{~Hz}$ passing through the water Cerenkov detector and therefore about $1 \mathrm{~Hz}$ through the scintillation vessel. By localizing the muon track using information from the Cerenkov detector. and declaring (in software) a veto region around each track, these muons and the delayed beta radioactivity they induce should be strongly suppressed without introduction of significant deadtime. Of course, direct demonstration of this situation is important. and we will carry out in situ measurements of the cosmic ray backgrounds with a ton-scale test detector (see Section 4.6 ).

Stopping muons must also be considered. with $\mu^{-}$probably the most troublesome because of their ability to undergo nuclear capture. We estimate the stopping $\mu^{-}$rate in 900 tons of scintillator to be about $600 \mathrm{~d}^{-1}$. Of these. about 540 will undergo beta decay with a mean life of about $2 \mu$ s and most of the remaining 60 will be captured with the release of a single neutron. An entering muon veto signal should tag more than $99 \%$ of these events. while time and pulse height cuts should give another factor of at least 10 for the 
muon beta decays. Again, the background situation looks good, but more detailed calculations and measurements are called for.

Probably the most troublesome external background is the interaction of high energy muons in the rock creating a single high energy neutron directed toward the scintillator. The neutron energy required to penetrate the water shield is several hundred $\mathrm{MeV}$. If such a neutron enters the scintillator, the huge recoil proton pulse should allow its rejection. The worst (but unlikely) scenario is for the high energy neutron to lose most of its energy in the water (where the recoil protons would be below Cerenkov threshold) and then to enter the scintillator with an energy of a few MeV. Using the CERN Monte Carlo code GEANT, we plan to study this type of event to judge the rate of false correlated events which might be produced.

Internal radioactivity We expect some difficulties with backgrounds to arise from the inevitable internal radioactive contamination of the detector materials. Laboratory measurements are in progress at Drexel aimed at evaluating and controlling this source of background.

We have estimated the chance coincidence rate due to internal radioactivit $y$ in the Perry detector with the assumption of uranium and thorium levels in the scintillator of $1 \times 10^{-13} \mathrm{~g} / \mathrm{g}$ and in the water shield 4 times higher. In the positron channel, the singles rate above a threshold of $2 \mathrm{MeV}$ would be $0.3 \mathrm{~Hz}$. while in the neutron capture channel the rate above $4 \mathrm{MeV}$ would be $0.1 \mathrm{~Hz}$ (mostly due to thorium). In a $100-\mu s$ gate, therefore, the chance coincidence rate would be $r_{1} r_{2} \tau=0.3 \mathrm{~d}^{-1}$. With the requirement of a $<2 \mathrm{~m}$ spatial coincidence between the two time-correlated events, the accidental rate would be suppressed by an additional factor of $\sim 30$ to a negligible level. 


\subsection{A chlorine-loaded neutron capture scintillator}

\subsubsection{Introduction}

Although neutrons can be detected by any of the common hydrocarbon scintillators, the time required for thermalization and capture of a neutron in such a scintillator is rather long, typically $170 \mu \mathrm{s}$. Furthermore, the energy released in the capture event is only $2.22 \mathrm{MeV}$, the binding energy of the deuteron. For clean detection of antineutrino inverse beta decay events, it is necessary to limit the chance coincidence rate to a very small value. The rate of chance coincidences is given by $r_{1} r_{2} \tau$, where $r_{1}$ is the counting rate in the positron energy window, $r_{2}$ the rate in the neutron window and $\tau$ the width of the coincidence window. The low neutron capture energy in hydrocarbon scintillators leads to a large value of $r_{2}$, since radioactive backgrounds generally increase rapidly below $2.61 \mathrm{MeV}$ (the energy of the $\mathrm{ThC}^{\prime \prime}$ gamma ray), while. for good detection efficiency, the long capture time requires a rather large value of $\tau$ (typically $500 \mu \mathrm{s}$ ).

Our term neutron capture scintillator refers to one in which a substance with a large neutron capture cross section has been incorporated. In Table 2 we list most of the elements with large neutron capture cross sections likely to be useful in a liquid scintillator, as well as the MACRO scintillator (row 1). a typical hydrocarbon scintillator, for comparison.

In column 4 of the table we show a typical loading percentage for each neutron capturing element and, in column 8 , the resulting capture time. Although the capture cross section of chlorine is 1500 times lower than that of gadolinium. it can be loaded at concentrations 400 times larger by weight corresponding to 1800 times larger by number of atoms. The capture time in a $20 \%$ chlorine-loaded scintillator is therefore about $40 \mu \mathrm{s}$, comparable to that in a typical gadolinium-loaded scintillator.

Chlorine-loaded scintillators, moreover, offer the promise of good stabilit $y^{1}$. transparency and freedom from thorium impurities ${ }^{2}$, and are much less expensive than gadolinium-loaded scintillators ${ }^{3}$.

\footnotetext{
${ }^{1} \mathrm{Gd}$-loaded scintillators have been reported to be somewhat unstable, with the gadolinium salt at risk of precipitating.

"Because of geochemical similarities between the rare earths and the actinides, gadolinium salts may contain as much as $1 \mathrm{ppm}$ of thorium

${ }^{3}$ The principal supplier of Gd-loaded liquid scintillators has informed us the price of gadolinium concentrate $(0.25 \%)$ would not be lower than $\$ 23$ /liter, even in very large
} 


$\begin{array}{ccccccccc}\begin{array}{c}\text { Capture } \\ \text { element }\end{array} & \begin{array}{c}\text { Atomic } \\ \text { mass } \\ \text { (amu) }\end{array} & \begin{array}{c}\sigma \\ \text { thermal } \\ \text { (barns) }\end{array} & \begin{array}{c}\text { Conc. } \\ \text { (typical) } \\ \text { (wt \%) }\end{array} & \begin{array}{c}\text { Scint. } \\ (\mathrm{g} / \mathrm{cc})\end{array} & \begin{array}{c}\mathrm{N} \\ \text { (atom/cc) }\end{array} & \begin{array}{c}\text { Mean } \\ \text { tree path } \\ (\mathrm{cm})\end{array} & \begin{array}{c}\text { Capture } \\ \text { time } \\ \text { (microsec) }\end{array} & \text { Signature } \\ \mathrm{H} & 1.0079 & 0.333 & 14.87 & 0.87 & 7.73 \mathrm{E}+22 & 38.84 & 177 & 2.2 \mathrm{MeV} \text { gamma } \\ \mathrm{Li} & 6.941 & 71 & 5 & 0.88 & 3.82 \mathrm{E}+21 & 3.69 & 16.8 & \mathrm{t}+\text { alpha } \\ \mathrm{B} & 10.81 & 765 & 5 & 0.90 & 2.51 \mathrm{E}+21 & 0.52 & 2.4 & 0.5 \mathrm{MeV} \text { gamma } \\ \mathrm{Cl} & 35.453 & 33.5 & 20 & 1.01 & 3.43 \mathrm{E}+21 & 8.70 & 39.5 & 8.6 \mathrm{MeV} \text { gamma } \\ \mathrm{Cd} & 112.41 & 2450 & 1 & 0.88 & 4.72 \mathrm{E}+19 & 8.66 & 39.3 & 8.9 \mathrm{MeV} \text { gamma } \\ \mathrm{Gd} & 157.25 & 49000 & 0.05 & 0.88 & 1.69 \mathrm{E}+18 & 12.11 & 55.0 & 8 \mathrm{MeV} \text { gamma }\end{array}$

Table 2: Comparison of neutron capture scintillators.

A further advantage of a chlorinated scintillator for the Perry experiment is its $\sim 1 \mathrm{~g} / \mathrm{cm}^{3}$ density, which may simplify construction of a suitable container. The disadvantage of these scintillators is that none have been described in the literature, and, therefore, special development work is required. The next three sections describe progress at Drexel on development of a chlorine-loaded scintillator suitable for the Perry experiment.

\subsubsection{Performance goals}

The performance goals we have established for the Perry scintillator are listed in Table 3.

\begin{tabular}{|l|l|}
\hline Chlorine content & $>20 \%$ by weight \\
Scintillation yield & $>30 \%$ of anthracene \\
Attenuation length & $>12 \mathrm{~m}$ \\
Flash point & $>140^{\circ} \mathrm{F}$ \\
Specific gravity & $\sim 1.01$ \\
C and Th impurities & $<0.1 \mathrm{ppt}$ \\
Cost & $<\$ 2.50 /$ liter \\
Stability & $>5 \mathrm{yr}$ \\
\hline
\end{tabular}

Table 3: Performance goals for the Perry scintillator.

quantities. After dilution with, for example, MACRO scintillator $(\$ 1.25 /$ liter $)$, the total cost would be about $\$ 5.60 /$ liter. We expect our chlorine-loaded liquid scintillator to cost less than half as much. 


\subsubsection{Scintillation yield and optical transparency}

After an extensive search for chlorine-containing materials suitable for liquid scintillation use, we have selected a few of the most promising for intensive study. These compounds are listed in Table 4 in decreasing order with respect to their chlorine content together with several of their physical properties.

$\begin{array}{lccccccc}\text { COMPOUND } & \begin{array}{c}\text { Mol } \\ \text { wt. }\end{array} & \text { MP } & \text { BP } & \begin{array}{c}\text { Flash } \\ \text { pt }\end{array} & \rho & \begin{array}{c}\text { Refractive } \\ \text { index }\end{array} & \text { [Cl] } \\ & & \text { C } & \text { C } & \text { F } & \text { g/cc } & & \text { wt.\% } \\ \text { methylene chloride } & 84.9 & -97 & 40 & \text { none } & 1.325 & 1.424 & 83.6 \\ \text { 123-trichloropropane } & 147.4 & -14 & 156 & 180 & 1.387 & 1.482 & 72.3 \\ \text { 12-dichloroethane } & 99.0 & -35 & 83 & 60 & 1.256 & 1.445 & 71.7 \\ \text { chlorinated olefin } & 273.5 & \text { low } & \text { high } & >250 & 1.190 & & 49.3 \\ \text { 13-dichlorobenzene } & 147.0 & -24 & 172 & 146 & 1.288 & 1.546 & 48.3 \\ \text { 14-dichlorobenzene } & 147.0 & 54 & 173 & 150 & 1.241 & \text { solid } & 48.3 \\ \text { chlorobenzene } & 112.6 & -45 & 132 & 75 & 1.107 & 1.524 & 31.5 \\ \text { 2-chlorotoluene } & 126.6 & -36 & 158 & 117 & 1.083 & 1.525 & 28.0 \\ \text { 1-chloronaphthalene } & 162.6 & -20 & 112 & 250 & 1.194 & 1.632 & 21.8\end{array}$

Table 4: Candidate materials for chlorinated scintillators.

Scintillation yield The scintillation yield of each chlorinated scintillator is measured using a $20 \mathrm{~cm}^{3}$ sample in a small vial irradiated by a ${ }^{60} \mathrm{Co}$ gamma ray source and viewed by a $2^{\prime \prime}$ bialkali photomultiplier tube. A typical set of pulse height spectra for four chlorobenzene-loaded scintillators is shown in Fig. 14. Since the principal feature in these spectra is the unresolved Compton edges of the 1.17 and $1.33-\mathrm{MeV}{ }^{60} \mathrm{Co}$ gamma rays, we take as reference the channel number of the midpoint of the Compton edge. The four curves show the pulse height spectra for scintillators containing, respectively, $17.20 \%, 30 \%$, and $50 \%$ chlorobenzene (by volume). The first scintillation mixture (vial 35) has an efficiency known to be $80 \%$ that of anthracene. The addition of chlorobenzene results in decreased pulse height, but even at $50 \%$ (vial 50 ). the efficiency is still an excellent $50 \%$ of anthracene. For 
comparison, the MACRO scintillator has an efficiency of $45 \%$ of anthracene. Note that these scintillation mixtures contain naphthalene.

The corresponding pulse heights without naphthalene are considerably lower, as shown in Fig. 15. Including naphthalene in the scintillation mixture is clearly very desirable. Table 5 summarizes the efficiencies of the chlorobenzene scintillators.

\begin{tabular}{|c|c|c|}
\hline $\begin{array}{c}\text { chlorobenzene } \\
\text { conc. (\%) }\end{array}$ & $\begin{array}{c}\text { Efficiency w/o } \\
\text { naphthalene }\end{array}$ & $\begin{array}{c}\text { Efficiency with } \\
\text { naphthalene }\end{array}$ \\
\hline 0 & $80 \%$ & $80 \%$ \\
20 & $30 \%$ & $67 \%$ \\
30 & $26 \%$ & $61 \%$ \\
50 & $20 \%$ & $50 \%$ \\
\hline
\end{tabular}

Table 5: Chlorobenzene scintillator efficiencies (relative to anthracene).

At present, perhaps the most interesting chlorinated material is $1,2,3$ trichloropropane $(1,2,3-$ TCP). In Fig. 16 we show the scintillation efficiency of a number of 1.2.3-TCP-based scintillators as a function of the 1,2,3-TCP content and of the amount of naphthalene added. Reasonable efficiency can be obtained even without naphthalene for concentrations of $1,2,3$-TCP up to $\sim 30 \%$.

Optical attenuation Optical attenuation of the chlorinated solvents is being measured with the Drexel long path laser spectrophotometer. Attenuation results for 1,2,3-TCP are shown in Fig. 17.

The attenuation length in the region of $420-440 \mathrm{~nm}$ is about $6 \mathrm{~m}$, a value lower than the desired $12 \mathrm{~m}$. However, there are two reasons for optimism. First. the chlorinated material is only a fraction of the complete scintillator. In the case of $1,2.3$-TCP, the chlorine content is $72.3 \%$. Obtaining a $20 \%$ chlorine loading in the scintillator therefore requires a $1,2,3$-TCP concentration of only $.20 / .723=27.7 \%$. The partial attenuation length due to the chlorine material alone is therefore $6 / .277=21.7 \mathrm{~m}$, which is quite satisfactory. provided the remaining components of the scintillator are reasonably 


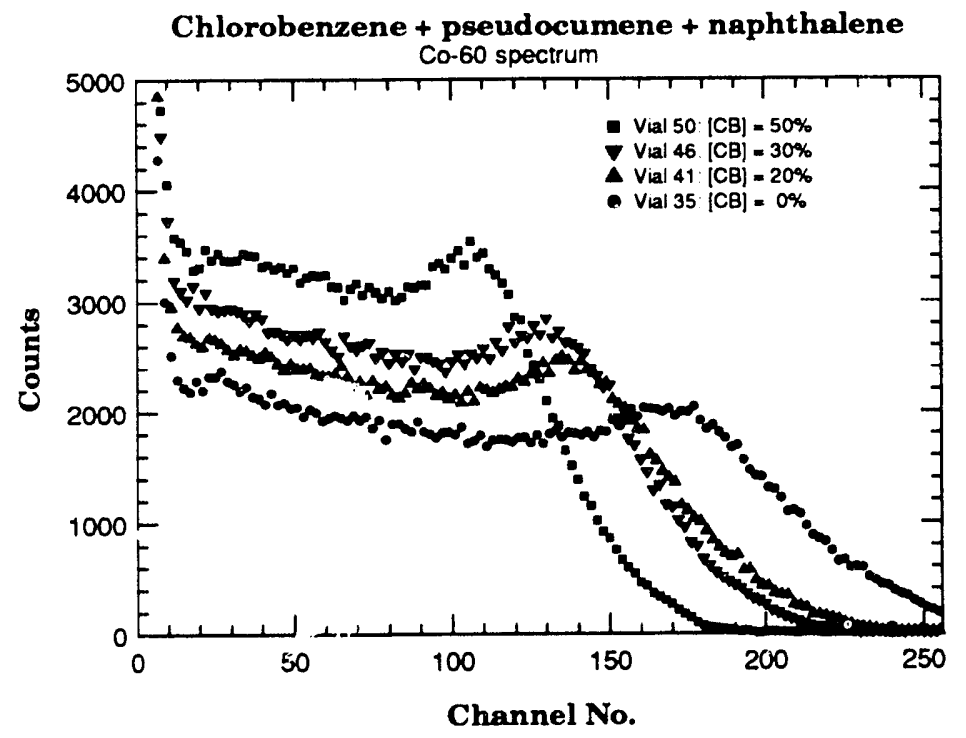

Figure 14: Chlorinated scintillator pulse height spectra. Vial 35 has an efficiency of $80 \%$ of anthracene, while the vial 50 efficiency is $50 \%$. Vials 41,46 and 50 contain $18 \%$ naphthalene.

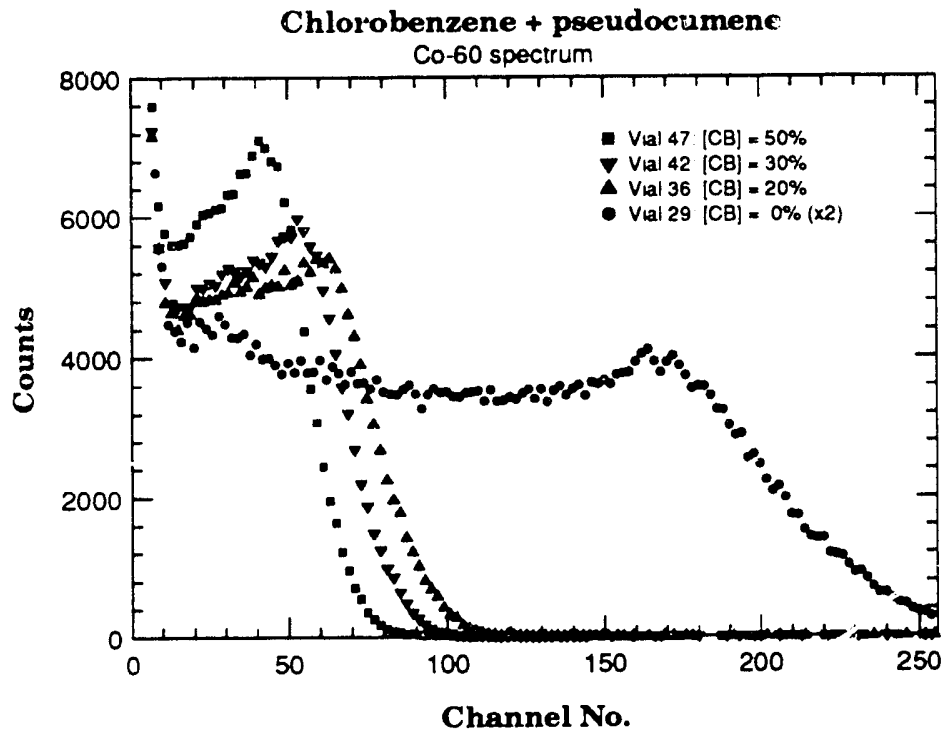

Figure 15: Chlorinated scintiliator pulse height spectra. No naphthalene has been added to these mixtures. Note the much lower pulse heights for the chlorinated scintillators without naphthalene compared to those in Fig. 14. 


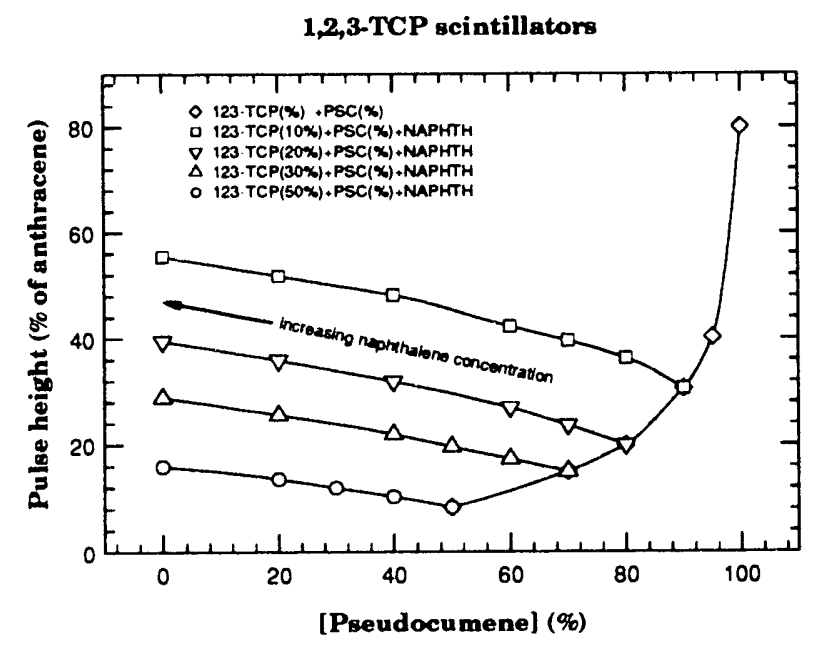

Figure 16: Scintillation efficiency of 1,2,3-trichloropropane scintillators

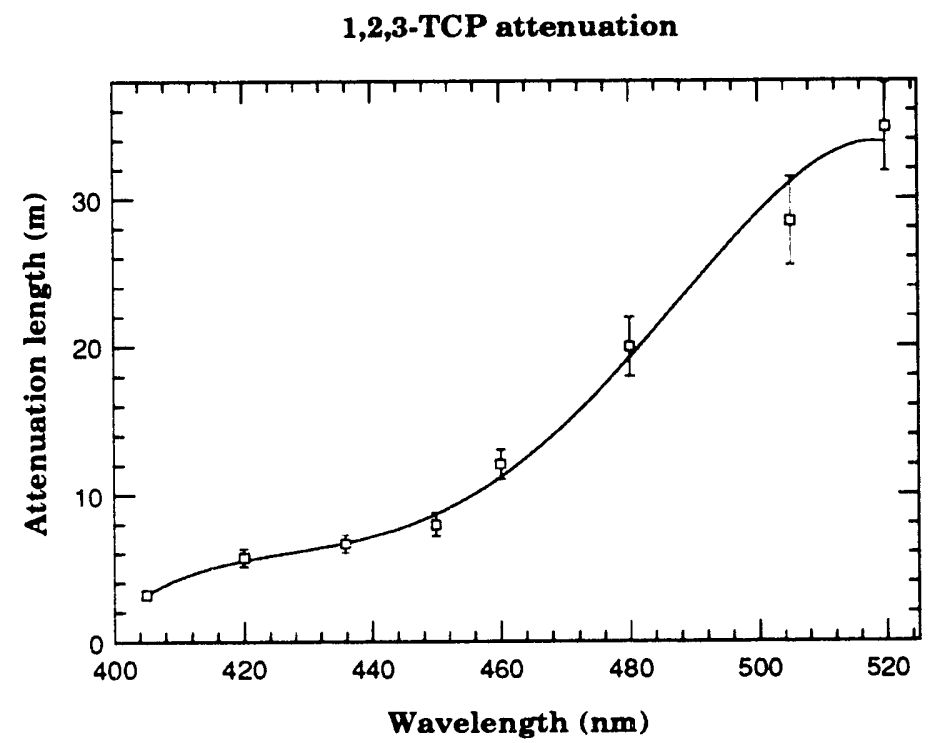

Figure 17: Optical attenuation for 1,2,3-trichloropropane. 
transparent. Second. we are testing a new wavelength shifter (not $B B Q$ ) which is known to be very fast and which shifts the PPO emission spectrum to the region near $490 \mathrm{~nm}$, where the attenuation lengths of most scintillation materials are much longer than at $430 \mathrm{~nm}$. For 1,2,3-TCP, for example, the attenuation length at $490 \mathrm{~nm}$ is more than $20 \mathrm{~m}$.

There are a number of problems remaining. The naphthalene samples we have tested so far have poor optical transmission. Unless better material can be found. we will have to forgo the very significant improvements in scintillation efficiency obtained by use of naphthalene. This problem is presently being addressed and the results at this moment are extremely encouraging.

\subsubsection{Outlook}

We consider completion of the development of a chlorinated scintillator suitable for the Perry experiment to be just a few months away. There are several very interesting candidate chlorinated solvents available, of which perhaps the most attractive at this time is 1,2.3-trichloropropane, with a very high chlorine content $(72.3 \%)$ and high flash point $\left(180^{\circ} \mathrm{F}\right)$, together with reasonable scintillation efficiency (especially with the addition of naphthalene) and attenuation length. This material is obtainable commercially in bulk quantities at reasonable cost.

Once a definite scintillator formulation has been developed, we plan to address the question of the content of radioactive impurities in the chlorinated scintillator. There seems to be no chemical reason why the chlorinated solvents should not be as free from uranium and thorium as is pseudocumene, which has been assayed in the $10^{-14} \mathrm{~g} / \mathrm{g}$ range for both $\mathrm{L}$ and $\mathrm{Th}$.

At that time. the cost of the scintillator will also be clearer. We will also be able to proceed with scintillation testing of ton quantities of the scintillator in the IMB facility. 


\subsection{Event generation and reconstruction}

As a first step in understanding and optimizing the potential performance of the Perry detector, we are modelling point events in one version of the detector using a method combining Monte Carlo techniques with ray tracing. The input parameters for the program, listed in Table 6, have not been optimized in any way, but have been chosen as an example of how the detector would perform in the least expensive experimental configuration, requiring the fewest modifications to the existing IMB detector. We have not included any photon collection enhancement techniques, such as Winston cones or wave shifter plates, nor have we considered optimizing the location of the existing PMT's or the possibility of using more and/or larger tubes. The results should therefore be taken as a minimal guaranteed performance level.

\begin{tabular}{|l|l|}
\hline Geometry' & 'standard IMB' + acrylic sphere \\
Radius of acrylic sphere & $6 \mathrm{~m}$ \\
Scintillation efficiency & $200 \mathrm{eV} /$ photon $=34 \%$ of anthracene \\
Scintiliator refractive index & 1.5 \\
Water refractive index & 1.335 \\
Scintillator attenuation length & $12 \mathrm{~m}$ \\
Water attenuation length & $20 \mathrm{~m}$ \\
PMT type & Hamamatsu R1408 $8^{\prime \prime}$ \\
PMT shape & oblate hemispheroid \\
PMT number & 2048 \\
PMT quantum efficiency & $20 \%$ \\
P.MT single pe time spread & $8 \mathrm{~ns}$ FWHM \\
Dynode collection factor & $50 \%$ \\
Event type & $8-\mathrm{MeV}$ random point in sphere \\
\hline
\end{tabular}

Table 6: Input parameters for point event reconstruction program.

Fig. 18 show the results of a ray tracing program for the above detector geometry: For source at distances greater than about $5.3 \mathrm{~m}$ from the center, some of the rays are totally reflected, resulting in some loss of intensity as 


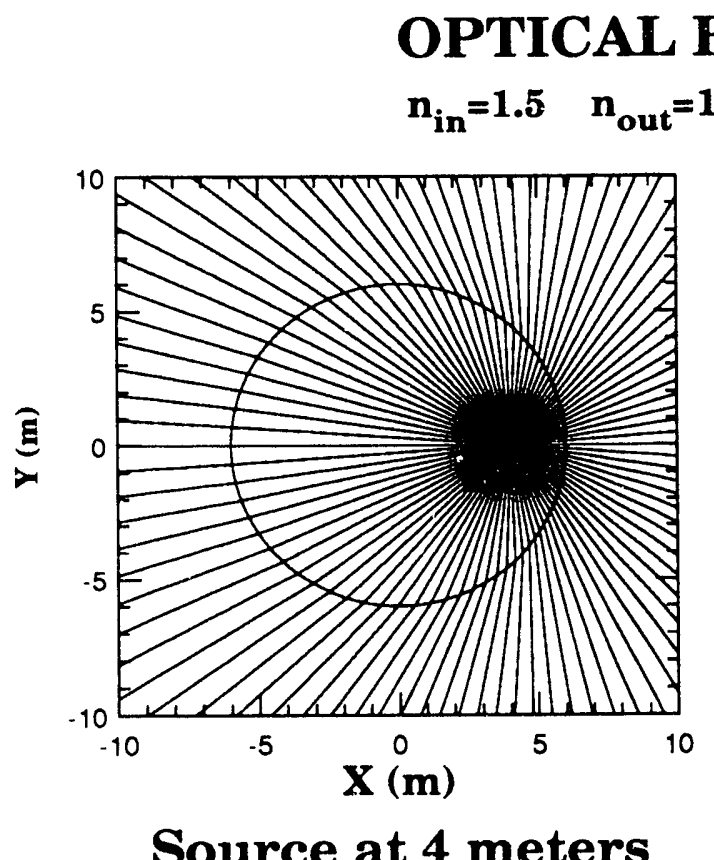

Source at 4 meters

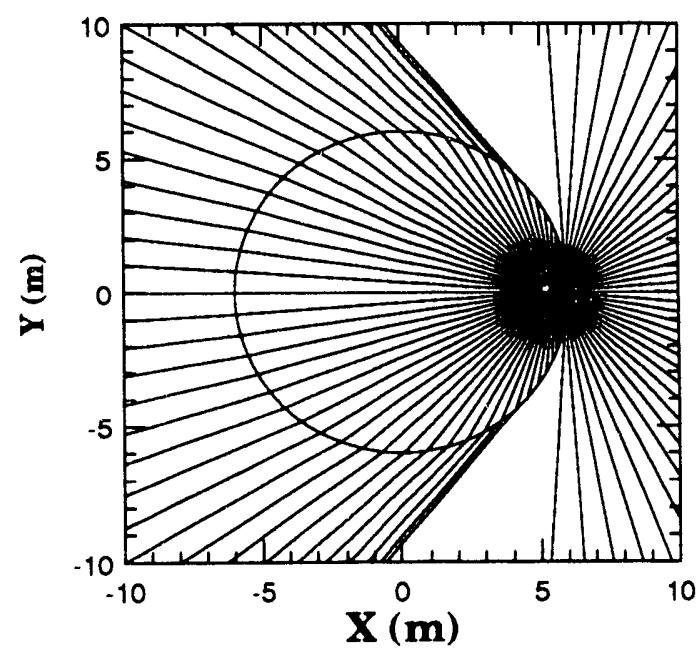

Source at 5.5 meters

Figure 18: Ray trace for point source in scintillator sphere. Note the total internal reflection for the event at $5.5 \mathrm{~m}$.

well as distortion of the event geometry. These optical effects have been fully taken into account by the event generation/reconstruction programs. The event distributions resulting from the reconstruction of $1008-\mathrm{MeV}$ point events in the detector are shown in Fig. 19. The mean number of photoelectrons produced ('PMT hits') is 63.4 or about 8 pe's $/ \mathrm{MeV}$. The reconstructed energy distribution has a standard deviation of $17.5 \%$, while the mean reconstruction error is about $40 \mathrm{~cm}$ with no events (out of 100) reconstructed more than $80 \mathrm{~cm}$ away from the true vertex. These figures seem reasonably good and indicate that even this rather modest detector configuration may well be adequate for the experiment.

Work is in progress using GEANT to extend these results to realistic events over the full reactor $\bar{\nu}_{\epsilon}$ spectrum. Preliminary results for reconstruction of positron events indicate a position reconstruction mean error of $66 \mathrm{~cm}$ with about $3 \%$ of the events having errors larger than $2 \mathrm{~m}$. 


\section{RECONSTRUCTION OF 100 8-MeV POINT EVENTS}
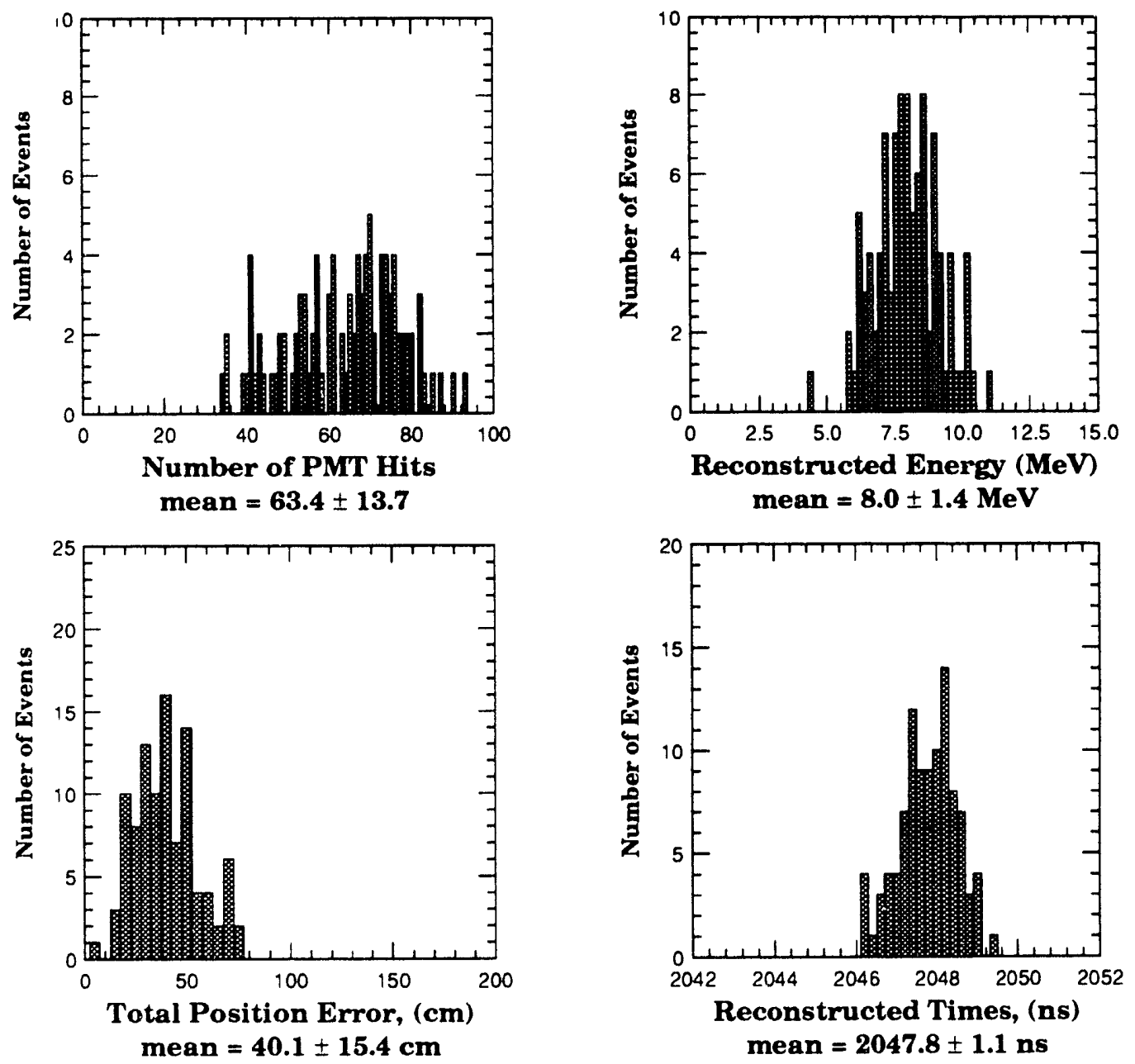

Figure 19: Results of reconstruction of $1008-\mathrm{MeV}$ point events. 


\subsection{In situ background tests}

Although laboratory studies are necessary to define the parameter space to explore in an experiment such as this one, the truth is that only on-site measurements can truly gauge the extent of background problems. For this reason. we are planning, in collaboration with members of the Irvine group, to undertake a set of measurements in the Morton salt mine facility. As a minimal set of measurements we plan the following:

- gamma ray and neutron surveys of the underground site;

- trace chemical analysis of the country rock;

- monitoring of the ambient radon and perhaps thoron levels;

- ultratrace analysis of the scintillator, water, and acrylic vessel;

- placement of an existing (at UCI's Savannah River site) 300-liter gadolinium-loaded liquid scintillator tank to monitor neutrons inside the water Cerenkov detector;

- installation of a roughly $1 \mathrm{~m}^{3}$ acrylic scintillator tank, which will be suspended in the water detector and run for about a week at each of several locations extending from the center nearly to the rock wall. This set of measurements will be particularly useful in defining the origin of singles backgrounds.

With data in hand from these measurements, we will be in a very good position to optimize the detector design without undue risk of unpleasant surprises. 


\section{$5 \quad$ Drexel Particle Physics Publications, 1991}

1. Cosmic Ray Search for Strange Quark Matter with the MACRO Detector, The MACRO Collaboration, Proc. Strange Quark Matter Workshop, (Denmark, May 1991).

2. A Search for Prompt Muons using the MACRO Detector at Gran Sasso, The MACRO Collaboration, Proc. 22nd Int'l. Cosmic Ray Conf,, (Dublin, Aug. 1991), HE 1.4-1.

3. Measurement of the Muon Decoherence Function with the MACRO Experiment at Gran Sasso, The MACRO Collaboration, Proc. 22nd Int'l. Cosmic Ray Conf., (Dublin, Aug. 1991), HE 4.2-6.

4. Search for Astrophysical Point Sources of Muons with the MACRO Detector, The MACRO Collaboration, Proc. 22nd Int'l. Cosmic Ray Conf., (Dublin, Aug. 1991), HE 4.3-6.

5. A Search for Sidereal Anisotropies with Underground Muons and an All-Sky Survey for Muon Point Sources with the MACRO Detector, The MACRO Collaboration, Proc. 22nd Int'l. Cosmic Ray Conf., (Dublin, Aug. 1991). HE 4.3-7.

6. Stellar Gravitational Collapse Detection by MACRO: Characteristics and Results, The MACRO Collaboration, Proc. 22nd Int'l. Cosmic Ray Conf., (Dublin, Aug. 1991), HE 5.3-17.

7. Search for Nuclearites with the MACRO Detector, The MACRO Collaboration. Proc. 22nd Int'l. Cosmic Ray Conf., (Dublin, Aug. 1991), HE 6.3-1.

8. Arrival Time Distributions of Very High Energy Cosmic Ray Muons in MACRO. The MACRO Collaboration, Proc. 22nd Int'l. Cosmic Ray Conf.. (Dublin, Aug. 1991), HE 4.2-7.

9. Study of the Primary Cosmic Ray Composition with the MACRO Experiment at Gran Sasso, The MACRO Collaboration, Proc. 22nd Int 'l. Cosmic Ray Conf.. (Dublin, Aug. 1991), OG 6.1-2. 
10. Measurement of Electromagnetic and TeV Muon Components of Extensive Air Showers by EAS-TOP and MACRO, The MACRO and EASTOP Collaborations, Proc. 22nd Int'l. Cosmic Ray Conf., (Dublin, Aug. 1991). HE 4.2-5.

11. Search for Nuclearites with the MACRO Detector, The MACRO Collaboration, Proc. Meeting APS Div. Part. and Fields, (Vancouver, Aug. 1991),

12. A Neutrino Oscillation Experiment Using the Perry Reactor and a Large Neutron Capture Scintillation Detector, Workshop on Future Experiments at the Fairport Facility, Boston, MA, June 1991.

13. Muon Astronomy with the MACRO Detector at Gran Sasso, The MACRO Collaboration, Bull. Am. Phys. Soc. 36, 1382 (1991).

14. Experimental Particle Astrophysics: A Report on the MACRO experiment, The MACRO Collaboration, Bull. Am. Phys. Soc. 36, 1697 (1991).

15. Recent Results and Future Prospects for the MACRO Experiment, The MACRO Collaboration, paper presented at the Int'l. School for Astroparticle Physics, Woodlands, TX, Jan. 1991. 


\section{References}

[1] The MACRO Collaboration, Proc. 21st Int'l. Cosmic Ray Conf., Adelaide, Australia, (1990).

[2] The MACRO Collaboration, Recent results from a magnetic monopole search with MACRO, Talk given at the Xth Moriond Workshop on New and Exotic Phen wena, Les Arcs, France, January 1990.

[3] The MACRO Collaboration, First results from the MACRO experiment, Invited paper given at the Washington D.C. meeting of the American Physical Society, April 16-19, 1990.

[4] The MACRO Collaboration, Study of penetrating cosmic ray muons and search for large-scale anisotropies at the Gran Sasso Laboratory, Physs. Lett. B249, 149 (1990).

[5] The MACRO Collaboration, Simultaneous observation of extensive air showers and deep-underground muons at the Gran Sasso Laboratory Phys. Rev. D42, 1396 (1990).

[6] The MACRO Collaboration, A study of charmed hadro-production with the MACRO detector at Gran Sasso, Proc. 22nd Int'l. Cosmic Ray Conf.. (Dublin. Aug. 1991), HE 1.4-1.

[i] The MACRO Collaboration, Measurement of the muon decoherence function with the MACRO experiment at Gran Sasso, Proc. 22nd Int'l. Cosmic Ray Conf., (Dublin, Aug. 1991), HE 4.2-6.

[8] The MACRO Collaboration, Search for astrophysical point sources of muons with the MACRO detector, Proc. 22nd Int'l. Cosmic Ray Conf., (Dublin. Aug. 1991). HE 4.3-6.

[9] The MACRO Collaboration, A search for sidereal anisotropies with underground muons and an all-sky survey for muon point sources with the MACRO detector, Proc. 22nd Int'l. Cosmic Ray Conf., (Dublin, Aug. 1991). HE 4.3-7. 
[10] The MACRO Collaboration, Stellar gravitational collapse in MACRO: characteristics and results, Proc. 22nd Int'l. Cosmic Ray Conf., (Dublin, Aug. 1991), HE 5.3-17.

[11] The MACRO Collaboration, Search for nuclearites with the MACRO detector. Proc. 22nd Int'l. Cosmic Ray Conf., (Dublin, Aug. 1991), HE 6.3-1.

[12] The MACRO Collaboration, Study of primary cosmic ray composition with the MACRO experiment at Gran Sasso, Proc. 22nd Int'l. Cosmic Ray Conf., (Dublin, Aug. 1991), OG 6.1-2.

[13] D.J. Ficenec, A search for magnetic monopoles and other supermassive charged particles, Ph.D. thesis, Boston University, (1990).

[14] D. Levin, Analysis of deep underground multiple muons and the cosmic ray composition above $10 \mathrm{TeV} /$ nucleus, Ph.D. thesis, Boston University, (1990).

[15] J. Petrakis, Muon astronomy with a large underground detector, Ph.D. thesis. Indiana University, (1990).

[16] The MACRO Collaboration, The MACRO detector at the Gran Sasso Laboratory, Nucl. Instrum. Meth. A264, 18 (1988).

[17] M.S. Turner, E.N. Parker and T.J. Bogdan, Magnetic monopoles and the survival of galactic magnetic fields, Phys. Rev., D26, 1296 (1982).

[18] S.P. Ahlen. T.M. Liss, C. Lane and G. Liu, Scintillation from slow protons: A probe of monopole detection capabilities, Phys. Rev. Lett. 55 (2), $181(1985)$.

[19] D.J. Ficenec, S.P. Ahlen, A.A. Marin, J.A. Musser and G. Tarlé, Observation of electronic excitation by extremely slow protons with applications to the detection of supermassive charged particles, Phys. Rev., D36. 311-314. (1987).

[20] X. Wen and E. Witten, Electric and magnetic charges in superstring models. Nucl. Phys., B261, 651 (1985). 
[21] (i. Zacek $t$ al. Neutrino-oscillation experiments at the Gösgen nuclear power reactor. Phys. Rev. D34, 2621 (1986). 

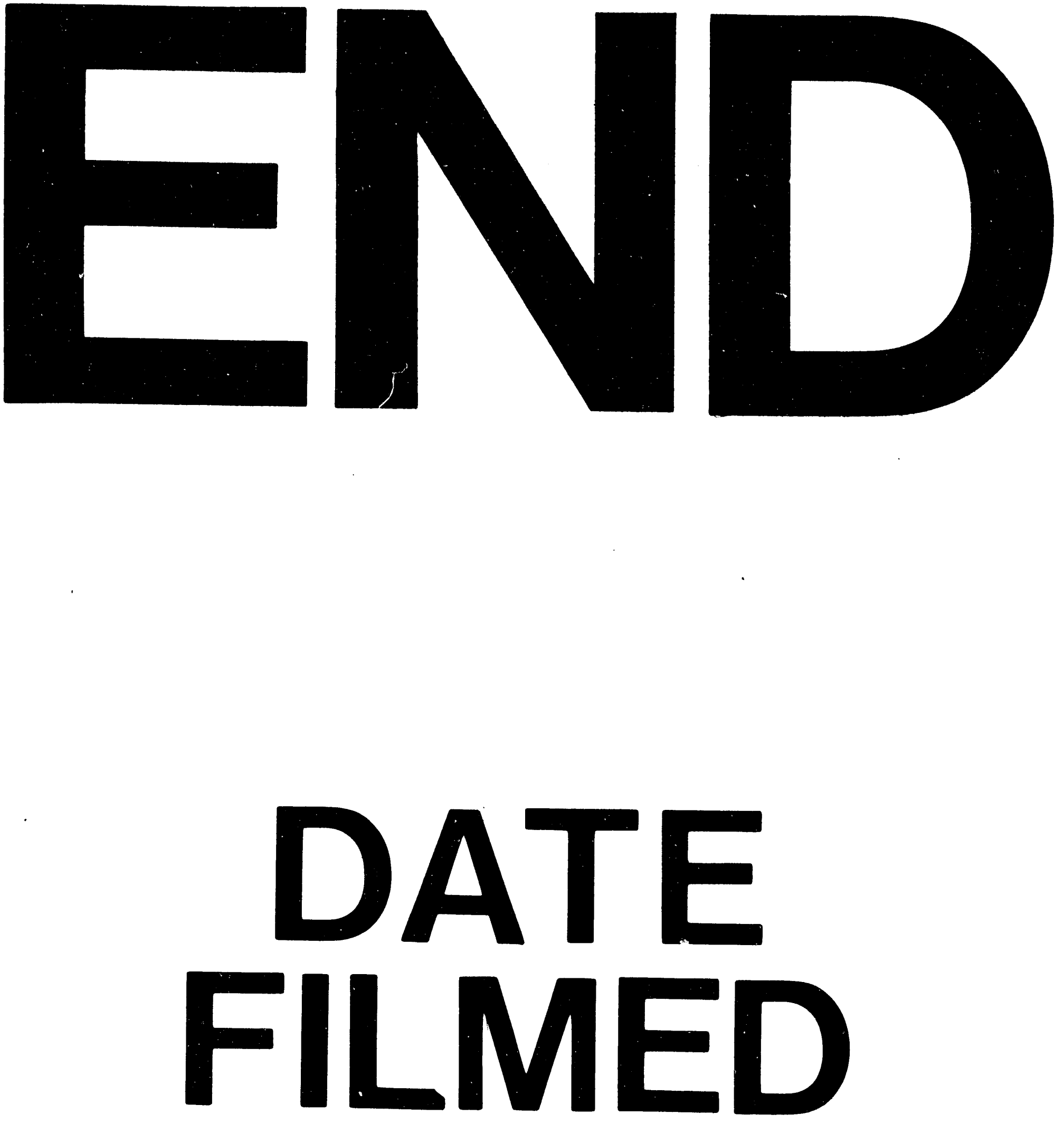

I

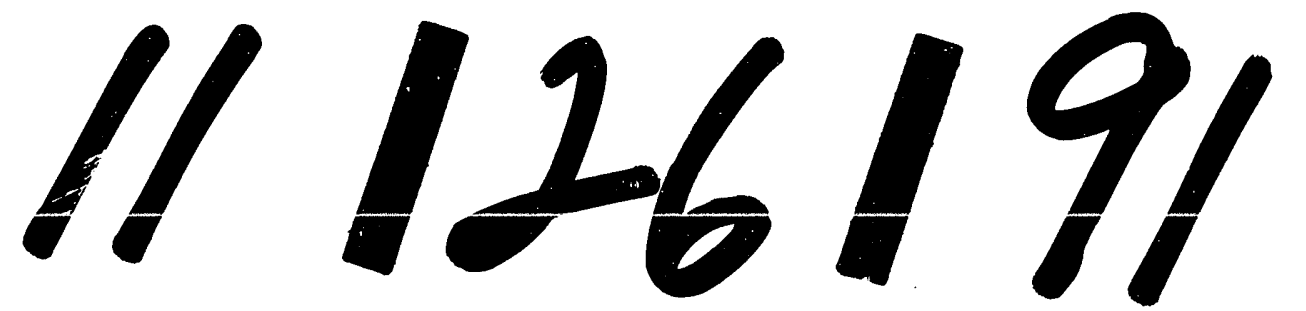

$=1$ 
